ARTICLE

Received 3 Jul 2015 | Accepted 29 Nov 2015 | Published 2 Feb 2016

DOI: $10.1038 /$ ncomms10336

OPEN

\title{
Sequential domain assembly of ribosomal protein S3 drives 40S subunit maturation
}

Valentin Mitterer ${ }^{1, \star}$, Guillaume Murat ${ }^{2, \star}$, Stéphane Réty $^{3}$, Magali Blaud $^{3}$, Lila Delbos ${ }^{3}$, Tamsyn Stanborough $^{1}$, Helmut Bergler ${ }^{1}$, Nicolas Leulliot ${ }^{3}$, Dieter Kressler ${ }^{2} \&$ Brigitte Pertschy $^{1}$

Eukaryotic ribosomes assemble by association of ribosomal RNA with ribosomal proteins into nuclear precursor particles, which undergo a complex maturation pathway coordinated by non-ribosomal assembly factors. Here, we provide functional insights into how successive structural re-arrangements in ribosomal protein $\mathrm{S} 3$ promote maturation of the $40 \mathrm{~S}$ ribosomal subunit. We show that $\mathrm{S} 3$ dimerizes and is imported into the nucleus with its $\mathrm{N}$-domain in a rotated conformation and associated with the chaperone Yar1. Initial assembly of S3 with $40 \mathrm{~S}$ precursors occurs via its $\mathrm{C}$-domain, while the $\mathrm{N}$-domain protrudes from the $40 \mathrm{~S}$ surface. Yar1 is replaced by the assembly factor Ltv1, thereby fixing the S3 $\mathrm{N}$-domain in the rotated orientation and preventing its $40 \mathrm{~S}$ association. Finally, Ltv1 release, triggered by phosphorylation, and flipping of the $\mathrm{S} 3 \mathrm{~N}$-domain into its final position results in the stable integration of S3. Such a stepwise assembly may represent a new paradigm for the incorporation of ribosomal proteins.

\footnotetext{
${ }^{1}$ Institut für Molekulare Biowissenschaften, Universität Graz, Humboldtstrasse 50, 8010 Graz, Austria. ${ }^{2}$ Unit of Biochemistry, Department of Biology, University of Fribourg, Chemin du Musée 10, CH-1700 Fribourg, Switzerland. ${ }^{3}$ Laboratoire de Cristallographie et RMN Biologiques, UMR CNRS 8015 , Université Paris Descartes, Sorbonne Paris Cité, Faculté de Pharmacie, 75006 Paris, France. ${ }^{\star}$ These authors contributed equally to this work. Correspondence and requests for materials should be addressed to N.L. (email: nicolas.leulliot@parisdescartes.fr) or to D.K. (email: dieter.kressler@unifr.ch) or to B.P. brigitte.pertschy@uni-graz.at).
} 
T he assembly of ribosomal RNA (rRNA) and ribosomal proteins (r-proteins) into eukaryotic ribosomes is a highly complex, multi-step process, which requires the coordinated action of over 200 assembly factors and takes successively place in the nucleolus, nucleoplasm and cytoplasm. Most of the $\sim 80$ r-proteins are assembled co-transcriptionally with a precursor rRNA, forming the large 90S particle (also termed SSU processome) that is subsequently separated into precursors of the large 60S (LSU) and the small 40S ribosomal subunit (SSU; reviewed in refs 1-4). Although most non-ribosomal 40S assembly factors present in the SSU processome leave the biogenesis pathway again in the nucleus, a few factors (namely Ltv1, Enp1, Pno1, Nob1, Dim1, Tsr1, Rio2, Hrr25, Fap7 and Rrp12) accompany the pre-ribosomal 40S particle to the cytoplasm. After fulfilling their distinct tasks, they are progressively released and recycled back to the nucleus in a hierarchical order that is not entirely resolved so $\mathrm{far}^{5-15}$. Cytoplasmic 40S maturation events include the final rRNA processing step of $20 \mathrm{~S}$ pre-rRNA into mature $18 \mathrm{~S}$ rRNA by the endonuclease Nob1 (refs 16-18), which takes place during a translation-like cycle in 80 S-like ribosomes after the transient joining of mature $60 \mathrm{~S}$ subunits ${ }^{10,19,20}$.

A crucial structural reorganization within the $40 \mathrm{~S}$ head domain, occurring before $20 \mathrm{~S}$ pre-rRNA cleavage, involves the r-protein S3 (Rps3) and leads to the formation of the characteristic beak structure, shaped by protrusion of $18 \mathrm{~S}$ rRNA helix 33 (ref. 8). Rps3 forms a ternary complex with the assembly factors Ltv1 and Enp1, which is salt-extractable from preribosomal particles ${ }^{8}$. Cryo-electron microscopy and crosslinking data revealed the position of Ltv1 and Enp1 on the pre-40S solvent side ${ }^{9,21}$. Within this complex, Ltv1 presumably adopts an elongated shape bridging the gap between the $40 \mathrm{~S}$ head (18S rRNA helix 41) and shoulder (18S rRNA helix 16) regions, and thereby preventing the opening of the mRNA entry channel. Beak formation coincides with the phosphorylation-dependent release of Ltv1 by the kinase Hrr25, which is supposed to be the initial step in cytoplasmic $40 \mathrm{~S}$ maturation $7,8,15$. During these remodelling steps, the initially weakly associated, salt-extractable Rps3 adopts its final position and becomes stably bound to the $40 \mathrm{~S}$ particle ${ }^{8}$; however, the mechanistic basis for this stable incorporation of Rps3 has remained elusive.

We have previously shown that, before its assembly into pre-ribosomal particles, Rps3 is associated with a specific chaperone, the ankyrin-repeat protein Yar1 (ref. 22). Recently, the co-crystal structure containing Rps3 and Yarl revealed the organization of this complex ${ }^{23}$. Rps3 is composed of two globular domains (hereafter referred to as Rps3 $\mathrm{N}$ - and C-domain) followed by an unstructured C-terminal tail. The K homology $\mathrm{N}$-domain of Rps3 binds to the central, structured core of Yar1 containing four ankyrin repeats. Compared to ribosome-bound Rps3, the structure of Rps3 in the Rps3/Yar1 complex shows two radical differences, which were both interpreted as non-physiological conformations formed as artefacts of crystallization $^{23}$ : (1) the Rps3 N-domain is rotated by $\sim 180^{\circ}$ relative to the $\mathrm{C}$-domain, and (2) the Rps3 $\mathrm{C}$-domain dimerizes with a second Rps3 C-domain by domain swapping, thereby forming a tetrameric Rps3/Yar1 complex.

In this study, we report the solution structure of the Rps3/Yar1 complex by small-angle X-ray scattering (SAXS), revealing that dimerization of the Rps3 C-domain and the relative orientation of the $\mathrm{N}$ - versus the $\mathrm{C}$-domain of Rps3 are not crystallization artefacts, but also occur in solution. Further, we show that Yar1-bound, non-ribosome-associated Rps3 also dimerizes in vivo. We demonstrate that Ltvl replaces Yarl as binding partner of the Rps3 $\mathrm{N}$-domain, thereby preventing premature $40 \mathrm{~S}$ incorporation of this domain. Initial assembly of Rps3 into pre-ribosomal particles is presumably mediated by its C-domain, thus, resolving the dimeric structure. A two-step mechanism, involving phosphorylation of Ltv1 and formation of critical contacts among Rps3, rRNA and the r-protein Rps20, eventually leads to the cytoplasmic release of Ltvl and hence the stable integration of the Rps3 N-domain into 40S subunits. Taken together, we have unveiled the intricate mechanisms regulating the stable assembly of Rps3, which promotes the final steps of cytoplasmic $40 \mathrm{~S}$ maturation.

\section{Results}

Rps3/Yar1 solution structure reveals Rps3 dimerization. Before assembly with ribosomal subunit precursors in the nucleus, Rps3 is bound by its specific chaperone Yar1, but how this interaction affects Rps3 incorporation into 40S precursors is currently unknown. To understand the molecular details of the transition from Yar1-bound Rps3 to ribosome-bound Rps3, we undertook a structural investigation of the Rps3/Yar1 complex in solution.

The crystal structure of the Rps3/Yar1 complex showed that the concave ankyrin-repeat surface of Yar1 binds the N-terminal $\alpha$-helix of Rps3 (residues 14-29), while the Rps3 C-domain is in contact with the second $\alpha$-helix of the fourth ankyrin-repeat ${ }^{23}$. In vitro binding assays confirmed that the very $\mathrm{N}$-terminal region of Rps3 mediates the interaction with the ankyrin-repeat core domain of Yar1 (Supplementary Fig. 1). To address how the complex is organized in solution, thereby avoiding crystallization artefacts, and to model the N- and C-terminal extensions of Yar1 and Rps3 missing in the crystal structure (corresponding to $\sim 30 \%$ of the residues), we determined the solution structure of the complex by SAXS. The data obtained for the Rps3 $\mathrm{N}$-domain (1-95)/Yar1 core (8-153) complex showed good agreement with a model constructed from the crystal structure (Fig. 1a; Supplementary Fig. 2a, middle panels; Supplementary Table 1). SAXS model structures of full-length Yar1 (Yar1-FL) confirmed the presence of unstructured extensions at the $\mathrm{N}$ and $\mathrm{C}$ termini; moreover, using this extended Yar1 structure, we could construct the Rps3 N-domain/Yar1-FL complex (Fig. 1a; Supplementary Fig. 2a and Supplementary Table 1). Next, we recorded SAXS data of the complex between the full-length Yar1 and Rps3 proteins. Surprisingly, we noticed that the molecular weight of the complex, as determined from the SAXS data, was twice as high as the sum of the calculated molecular weights of the individual components (Supplementary Table 1), indicating that the Rps $3 /$ Yarl complex dimerizes. The fact that dimerization of the Rps3/Yar1 complex was only observed when full-length Rps3 was present, but not when the Rps3 C-domain was absent, suggested that the dimerization is mediated by the Rps3 C-domain. In the crystal structure, the Rps3/Yar1 complex is found in tetrameric arrangements (Supplementary Fig. 2b) ${ }^{23}$. To determine the conformation present in solution, we built three initial models representing the three dimeric forms of the Rps $3 / Y a r 1$ complex that can be extracted from the crystal structure, corresponding to the dimers between complex 1 in Supplementary Fig. $2 \mathrm{~b}$ and complexes 2, 3 and 4, respectively (denoted as 1-2 dimer, 1-3 dimer and 1-4 dimer hereafter; Supplementary Fig. $2 \mathrm{~b}, \mathrm{c})$. The 1-4 dimer exhibited the conformation that fitted best to the SAXS data (Fig. 1b,c; Supplementary Fig. 2b,c). Interestingly, dimerization in this structure involves domain swapping of the two C-terminal $\beta$-strands of one Rps3 molecule with the equivalent $\beta$-strands of the opposing Rps3 molecule (Fig. 1c) ${ }^{23}$. Swapping of these two $\beta$-sheets restores a similar fold of the Rps3 C-domain as present in the ribosome-bound structure (Fig. 1d ${ }^{24}$. However, the relative orientation between the Rps3 C- and $\mathrm{N}$-domains in the Rps3/Yar1 complex markedly differs from the Rps3 conformation in the mature ribosome, with the $\mathrm{N}$-domain 
a
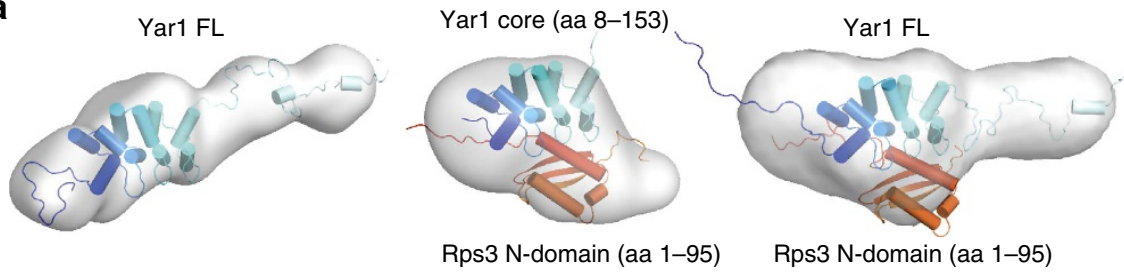

Rps3 N-domain (aa 1-95) Rps3 N-domain (aa 1-95)

b

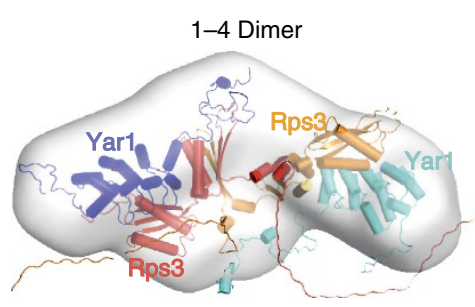

C

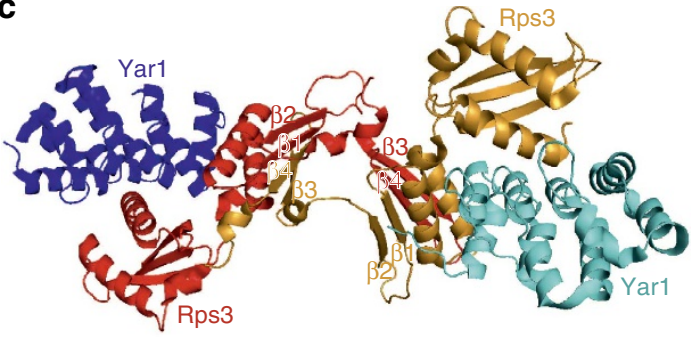

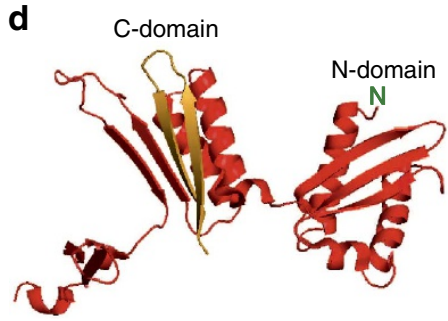

Rps3-domain swapped

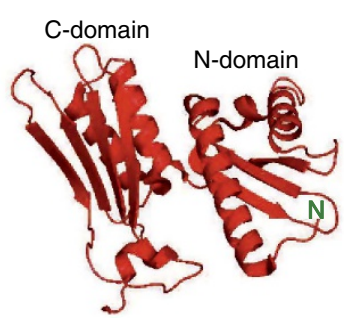

Rps3-40S bound

Figure 1 | The Rps3 C-domain is rotated relative to the $\mathbf{N}$-domain and dimerizes by domain swapping. (a) Model fit into a representative SAXS envelope for full-length Yar1 (FL), Rps3 N-domain (1-95)/Yar1 core (8-153) complex, Rps3 N-domain/Yar1-FL complex. Yar1 is coloured from blue (N terminus) to light blue ( $\mathrm{C}$ terminus), Rps3 is coloured in orange. (b) Tetrameric arrangement of full-length Rps3/Yar1 complex. A representative envelope and the best fitting model of the dimeric conformations shown in Supplementary Fig. 2c extracted from the crystal structure (1-4 dimer) are shown. (c) Crystal structure of the 1-4 dimer (Protein Data Bank (PDB) 4BSZ). The same colours are used as in (b). The four $\beta$-sheets of the Rps3 C-domain were numbered to highlight that $\beta$-sheets 3 and 4 are swapped between the two Rps3 molecules. (d) Rps3 structure extracted from (c) with completion of the fold by the two swapped $\beta$-sheets (left panel). Domain swapping restores a similar fold of the C-domain as found in ribosome-bound Rps3 (PDB 3U5C, right panel). Comparing the two Rps3 conformations, the $\mathrm{N}$-domain is rotated by $\sim 180^{\circ}$ relative to the $\mathrm{C}$-domain.

rotated by $\sim 180^{\circ}$ (Fig. 1d). Taken together, our experimental SAXS data reveal that Rps3 dimerization by domain swapping occurs in solution and moreover indicate that the relative orientation between the Rps $3 \mathrm{~N}$ - and C-domains, which differs from the conformation in the ribosome by a $\sim 180^{\circ}$ rotation (Fig. 1d $)^{24}$, is not a crystallization artefact and might be physiologically relevant.

Rps3 dimerizes in vivo. We previously conducted a random PCR mutagenesis of RPS3 and screened for mutants showing a synthetic growth defect in combination with the yar $1 \Delta$ mutant ${ }^{22}$. Five mutants were isolated, each resulting in two to three amino acid exchanges in Rps3 (Supplementary Fig. 3a,b). Notably, four of these mutants contained one exchange in residues within the C-domain forming contacts involved in domain swapping. On the basis of these genetic data, we considered that domain swapping and consequently dimerization of Rps3 might also occur in vivo.

Next, we aimed to test whether Rps3 forms dimers in the Rps3/Yar1 complex in vivo, applying a strategy adapted to the low amounts of endogenous complex that can be purified from yeast cells. To this end, we used a diploid yeast strain with tandem affinity purification tag (TAP-tag) fusions of both YAR1 alleles, and a Flag-tag fusion of one RPS3 allele (while the second RPS3 allele remained untagged; experimental scheme in Fig. 2a).
We performed TAP of Yar1, followed by Flag purification of Rps3. If Rps3 occurs as a monomer in complex with Yar1, only Yar1-calmodulin-binding protein (Yar1-CBP) and Rps3-Flag should be recovered after this purification procedure (Fig. 2a, left panel). If, however, Rps3 dimerizes, Yar1-CBP and both Rps3-Flag and untagged Rps3 should be recovered (Fig. 2a, right panel). Indeed, we detected not only Rps3-Flag but also untagged Rps3 in the purification, confirming dimerization of Rps3 within the Rps3/Yar1 complex (Fig. 2b, strain 2). In addition, we tested whether two Yar1 molecules are present in the Rps3/Yar1 complex, as suggested by the SAXS analyses. To this end, we used a diploid strain with one YAR1 allele TAP-tagged and the second allele untagged, together with one Flag-tagged and one untagged RPS3 allele. In this setup, again both Rps3-Flag and untagged Rps3 were purified, while surprisingly only Yar1-CBP and no untagged Yar1 was found in the eluate, suggesting that in vivo, only one Yar1 is bound to an Rps3 dimer (Fig. 2b, strain 1). To further confirm this observation, we performed the inverse experiment, using a strain with both RPS3 alleles TAP-tagged and one Flag-tagged, and one untagged YAR1 allele (Fig. 2c,d). In this setup, Rps3-containing complexes (that is, ribosomes, pre-ribosomal particles and Rps3/Yar1 complex) are purified in the first step. As expected, both Yar1-Flag and Yar1 were recovered after this purification step (Fig. 2d). However, after subsequent Flag purification, only Yar1-Flag, but no untagged Yar1 was eluted, suggesting only one Yar1 molecule is present in 
a

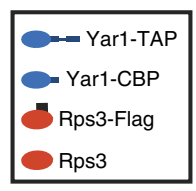

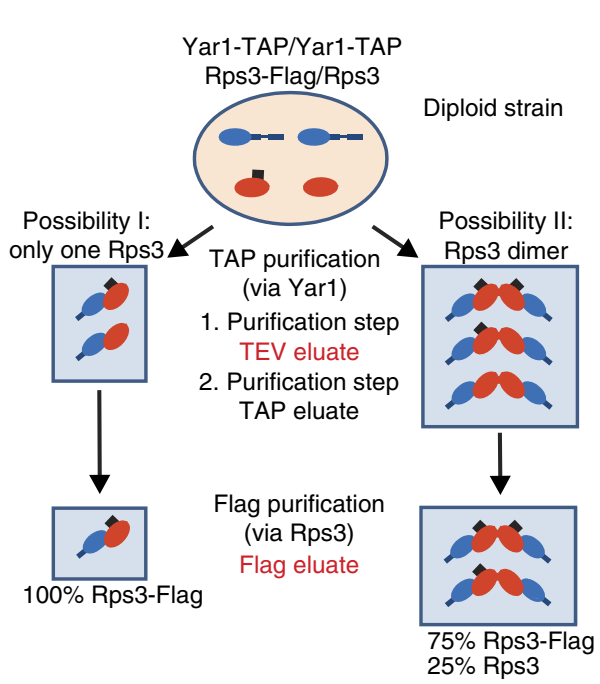

Yar1-TAP/Yar1-TAP

(via Yar1)

Purification step

TEV eluate

Purification step

TAP eluate

Flag purification (via Rps3)

Flag eluate

$25 \%$ Rps3
C

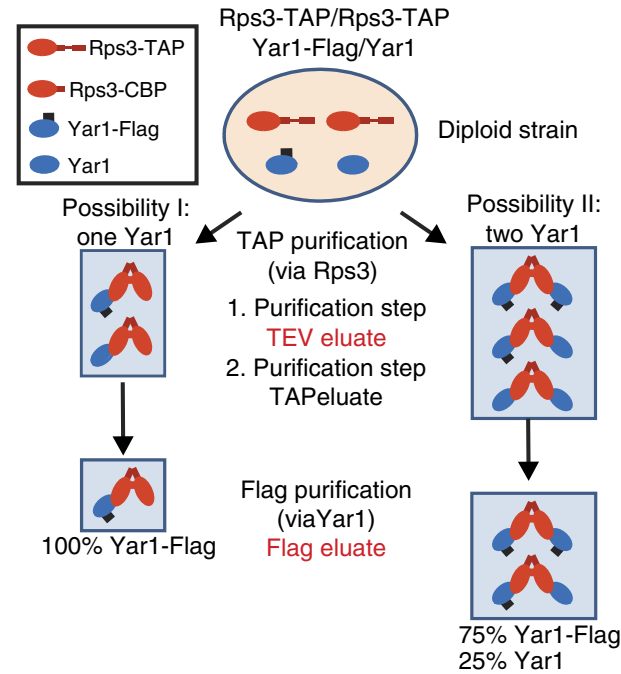

b

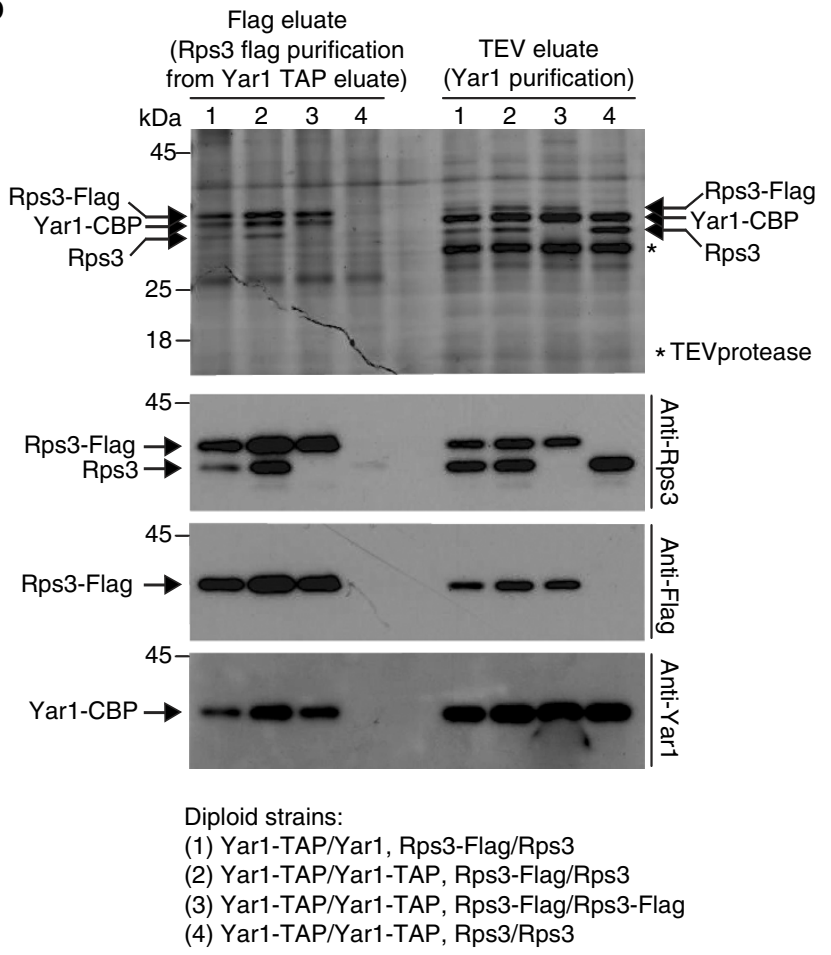

d
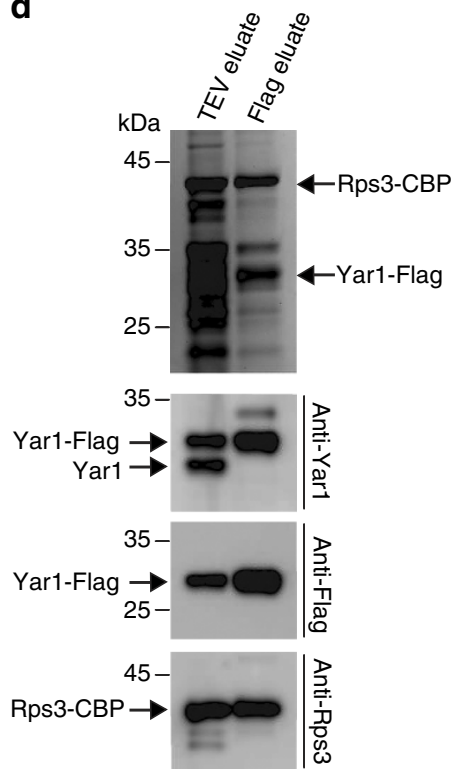

Diploid strain:

Rps3-TAP/Rps3-TAP,Yar1-Flag/Yar1

Figure 2 | Rps3 dimerizes in vivo. (a) Experimental scheme of the purification shown in (b). The TAP-tag is composed of a protein A part, a TEV cleavage site and a calmodulin-binding protein (CBP) moiety. First, Yar1 is purified via protein A and eluted by the TEV protease, releasing Yar1 fused to CBP (TEV eluate). Then, Yar1 is purified via the CBP tag and eluted (TAP eluate). Finally, Rps3-Flag contained in the eluate is purified. While only Yar1-CBP and Rps3Flag should be recovered in the case of a monomeric complex, the occurrence of dimeric complexes would also lead to co-purification of untagged Rps3. Fractions analysed in the experiment in (b) are indicated in red letters. (b) The indicated diploid strains (numbers 1-4) were subjected to the purification protocol outlined in (a). TEV eluates and Flag eluates were analysed by SDS-PAGE and Coomassie staining (upper part) as well as western blotting using the indicated antibodies (lower three panels). Note that using strains 1 and 2, both Rps3-Flag and untagged Rps3 are found in the Flag eluates, indicating dimerization of Rps3. In contrast, only one Yar1 was present in the complex, as revealed by the absence of untagged Yar1 in the TEV and Flag eluate of strain 1. When both RPS3 alleles were Flag-tagged (strain 3), only Flag-tagged Rps3 was found in the eluate, showing that the recovery of untagged Rps3 with strains 1 and 2 is not a consequence of proteolytic removal of the Flag-tag. Furthermore, no Rps 3 was recovered when both RPS3 alleles were untagged (strain 4). (c) Experimental scheme of the Rps3-TAP followed by Yar1-Flag purification shown in (d). (d) The diploid RPS3-TAP/RPS3-TAP YAR1-Flag/YAR1 strain was subjected to the purification protocol outlined in (c). TEV eluates and Flag eluates were analysed by SDS-PAGE and Coomassie staining (upper part) as well as western blotting (lower three panels). Note that in the first purification step (TEV eluate) both Yar1-Flag and Yar1 are co-purified, showing that both can form complexes together with Rps3. However, after subsequent purification of Yar1-Flag, no untagged Yar1 is recovered, corroborating that each complex contains only one Yar1 copy. 
each complex. We conclude that, in vivo, one Yarl is bound to an Rps3 dimer (see also Discussion).

Ltv1 replaces Yar1 as a binding partner of the Rps3 N-domain. Next, we aimed to further follow the ribosome assembly path of Rps3. Inspection of crosslinking data, and the pre-40S cryo-electron microscopy structure revealed that assembly of the Rps3 N-domain in its mature conformation would clash with binding of the $40 \mathrm{~S}$ assembly factor Ltv1 at helix 41 (around $18 \mathrm{~S}$ rRNA nucleotide C1490) $)^{9,21,24}$, suggesting that the Rps3 $\mathrm{N}$-domain should be displaced within pre-40S particles. Moreover, footprinting analyses revealed that in pre-40S particles, the nucleotides in helix 41 contacted by the $\mathrm{N}$ terminus of Rps3 are more reactive to chemical modification, further supporting that Rps3 is not bound at that site in pre-40S particles ${ }^{25}$.

These data, together with the fact that Yar1 binds to the Rps3 $\mathrm{N}$-domain, suggest that initial $40 \mathrm{~S}$ incorporation of Rps3 most likely occurs via its $\mathrm{C}$-domain, which represents the main rRNA-binding surface of Rps3 (refs 23,24). Considering that mature 40S subunits contain only one copy of Rps3, the second Rps 3 copy in the Rps3/Rps3/Yarl complex is presumably released upon ribosome assembly. Consistently, the amounts of Rps3 relative to other $\mathrm{r}$-proteins were equal in purified pre-ribosomal particles and mature $40 \mathrm{~S}$ subunits, suggesting that the stoichiometry of Rps3 remains the same during the course of pre-40S maturation (Supplementary Fig. 4a).

Since we were unable to detect significant amounts of Yar1 in purified pre-ribosomal particles and as Yar1 sediments in the soluble fraction of sucrose gradients ${ }^{22,26}$, Yar1 likely dissociates from Rps3 before, upon, or shortly after pre-ribosome assembly of Rps3. Hence, on pre-ribosomal particles, other assembly factors might bind the Rps3 $\mathrm{N}$-domain subsequently to Yar1. Rps3 was shown to form a salt-extractable complex with Ltvl and Enp1 in pre-40S particles ${ }^{8}$. Thus, we considered Ltv1 and Enp1 as candidates for successors of Yar1 in Rps3 N-domain binding.

Yeast two-hybrid $(\mathrm{Y} 2 \mathrm{H})$ analyses revealed a strong interaction between Rps3 and Ltv1 (Fig. 3a, right panel) ${ }^{27}$, while Enp1 did not interact with Rps3 (Fig. 3a, right panel). Moreover, Rps3 and Ltv1 showed a robust in vitro interaction (Supplementary Fig. $4 \mathrm{~b})^{7}$. Interaction domain mapping by $\mathrm{Y} 2 \mathrm{H}$ identified a large fragment (amino acids 15-95) of the Rps3 N-domain to be both necessary and sufficient for Ltv1 binding (Fig. 3a, right panel). Hence, Ltv1 and Yar1 both interact with the Rps3 $\mathrm{N}$-domain and have partially overlapping binding sites. To further assess whether Yarl and Ltvl compete for the same binding site on Rps3, we performed in vitro competition assays (Fig. 3b,c). Indeed, upon incubation of the Rps3/Yar1 complex, immobilized on Ni-NTA agarose via His6-Rps3, with purified Ltv1-Flag, Ltv1 bound to Rps3 and was present in the eluate, while Yar1 was released into the supernatant (Fig. 3b, lanes 4 and 10). In contrast, Enp1 was not able to bind Rps3 and compete Yar1 off (Fig. 3b, lanes 5 and 11). However, when Ltv1 was also present, a ternary Rps3/Ltv1/Enp1 complex was established, leading to Yar1 release (Fig. 3b, lanes 6 and 12). Next, we immobilized Rps3/Ltv1 complex on Ni-NTA agarose via His6-Rps3 and incubated the complex with purified Flag-Yar1. Although some Yar1 binding to Rps3 (Fig. 3c, lanes 3 and 4) and some Ltv1 release (Fig. 3c, lanes 7 and 8) was observed, Yarl was clearly less efficient in competing Ltv1 off Rps3 than Ltv1 was in competing Yarl off (Fig. 3b,c). We conclude that Ltv1 has a higher affinity for Rps3 than Yarl. Interestingly, a recent study indicates that in vitro Rps3 is efficiently loaded onto preribosomal particles only in the presence of Ltv1 (ref. 7). We suggest that the Rps3 $\mathrm{N}$-domain is handed over from the
Rps3/Yar1 complex to Ltv1 shortly before, during or shortly after Rps3 assembly. Eventually, Ltv1 needs to be released again in order to allow for the final incorporation of the Rps3 N-domain into the $40 \mathrm{~S}$ ribosomal subunit.

Ltv1 is released upon phosphorylation at S336, 339 and 342. Cytoplasmic release of Ltvl requires its phosphorylation by the kinase Hrr25 (CK1 $\delta / \varepsilon$ in human cells), and is a prerequisite for final 40 S maturation steps such as $20 \mathrm{~S}$ to $18 \mathrm{~S}$ rRNA processing, beak formation and the formation of $80 \mathrm{~S}$-like ribosomes ${ }^{7,8,15}$. To further characterize this event, we attempted to identify the target site(s) within Ltv1 that are phosphorylated by Hrr25. Alignment of the Ltv1 protein sequence from different species revealed a conserved serine/threonine-rich motif from amino acids 336 to 346 , resembling the consensus sequence recognized by CK1 (Fig. 4a) ${ }^{28,29}$. We substituted several different combinations of serines within this motif to alanine, and assessed the growth phenotypes of the resulting mutants (Supplementary Fig. 5a). The strongest growth defect was observed after combined substitution of the three serines S336, S339 and S342 to alanine. In contrast, substitution of the same serine residues to glutamate, which mimics phosphorylation, supported almost wild-type growth (Fig. 4b; Supplementary Fig. 5a). Moreover, overexpression of $\operatorname{Ltv1}(\mathrm{S} 336 \mathrm{~A} / \mathrm{S} 339 \mathrm{~A} / \mathrm{S} 342 \mathrm{~A})$, but not of Ltv1(S336E/S339E/S342E), resulted in a dominant-negative growth phenotype (Fig. 4c; Supplementary Fig. 5b). In vitro phosphorylation of Ltv1(S336A/S339A/S342A) by Hrr25 was clearly decreased compared to wild-type Ltv1, verifying these residues as the major Ltv1-phosphorylation sites (Fig. 4d; Supplementary Fig. 5c). While this manuscript was in preparation, the same residues were also reported by another study to be the main Ltv1-phosphorylation sites ${ }^{7}$. Ltv1 mutant protein with serine to alanine substitutions of all six serines from S336 to S346 (hereafter termed Ltv1(S6A)) was even less phosphorylated revealing that, in addition to S336, S339 and S342, also S344, S345 and S346 can be used as phosphorylation targets (Fig. 4d). However, the ltv1(S6A) mutant showed normal growth and no dominant-negative effect upon overexpression (Fig. $4 b, c)$, suggesting that the multiple mutations lead to a looser association with $40 \mathrm{~S}$ particles and allow the spontaneous dissociation of the protein without phosphorylation.

Next, we addressed whether cytoplasmic release of Ltv1(S336A/S339A/S342A) from pre-40S particles is impaired. Ltv1 has a cytoplasmic steady-state localization in wild-type cells (Fig. 5e), however, inhibition of nuclear export by leptomycin B (LMB) treatment of an LMB-sensitive $\mathrm{crm} 1$ mutant resulted in a substantial nuclear accumulation of Ltv1-green fluorescent protein (Ltv1-GFP) already after 10 min (Fig. 4e). A similar accumulation of Ltv1-GFP was also observed when translation was blocked before LMB treatment by cycloheximide (CHX) addition, indicating that the nuclear accumulation is mainly due to recycling and not to neosynthesis of Ltv1-GFP (Supplementary Fig. 5e). In contrast, Ltv1(S336A/S339A/S342A)-GFP remained cytoplasmic after $10 \mathrm{~min}$ of LMB treatment. Some nuclear accumulation was only detected after $60 \mathrm{~min}$, indicating a delay in recycling of Ltv1 back into the nucleus, hence suggesting a cytoplasmic pre-40S release defect (Fig. 4e; Supplementary Fig. 5e). Contrarily, the Ltv1(S6A) protein showed the same localization as wild-type Ltv1, indicating that cytoplasmic release was not impaired for this protein (Supplementary Fig. 5e).

The cytoplasmic release defect of the Ltvl(S336A/S339A/ S342A) phosphomutant was further confirmed by sucrose gradient centrifugation, which revealed increased levels of the mutated Ltvl sedimenting in the $40 \mathrm{~S}$ fractions of the gradient, whereas wild-type Ltv1 and Ltv1(S6A) were 

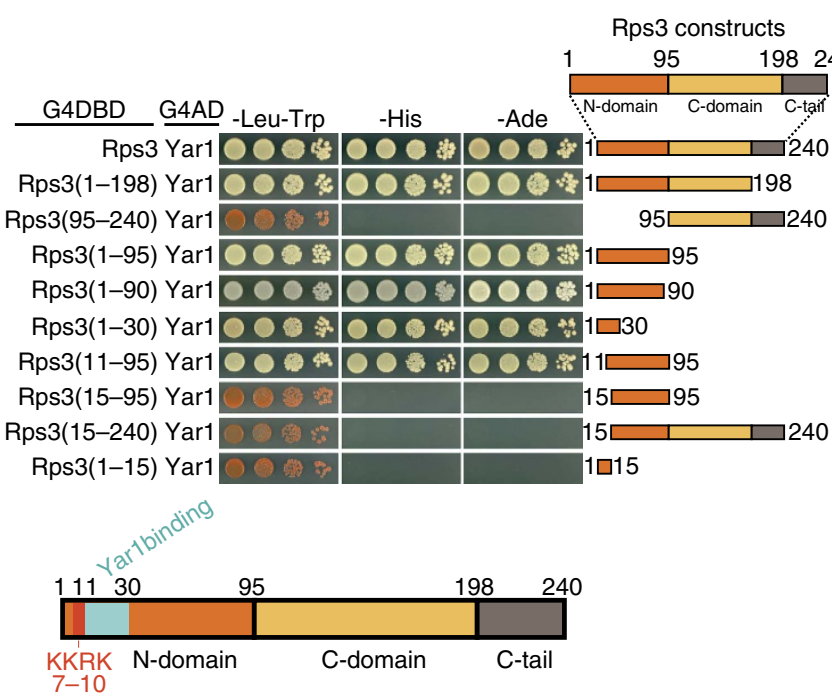

b

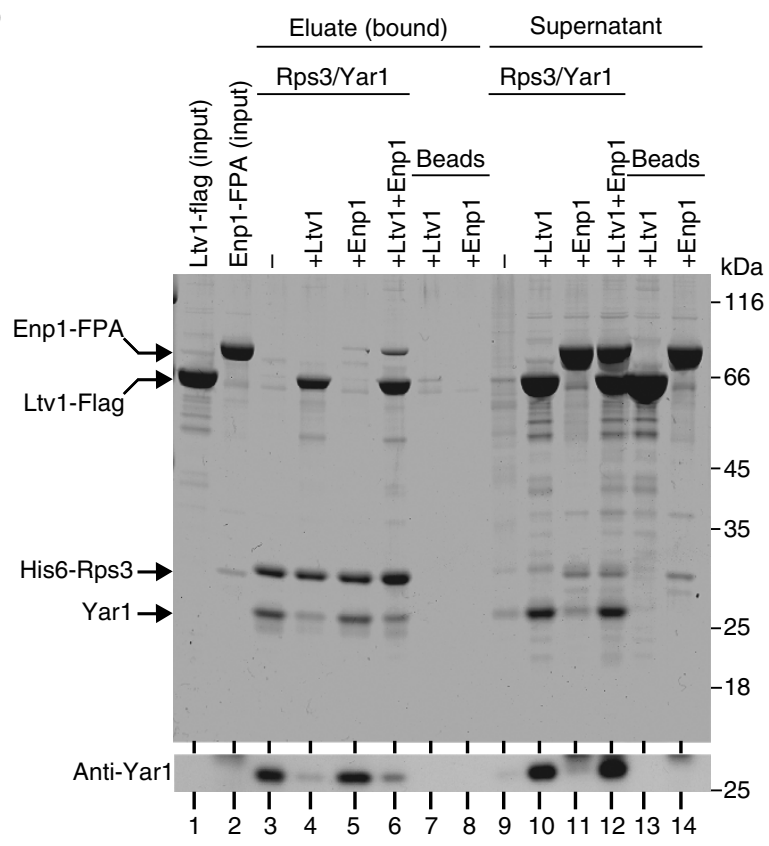

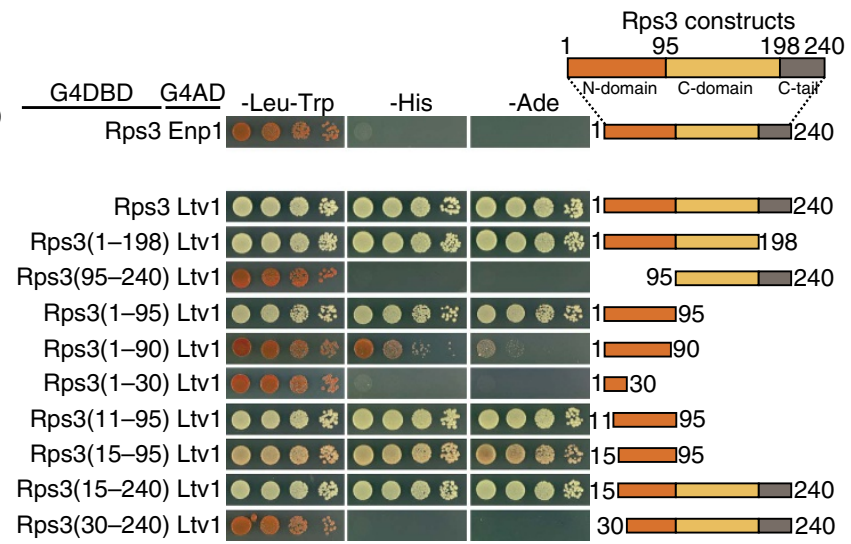
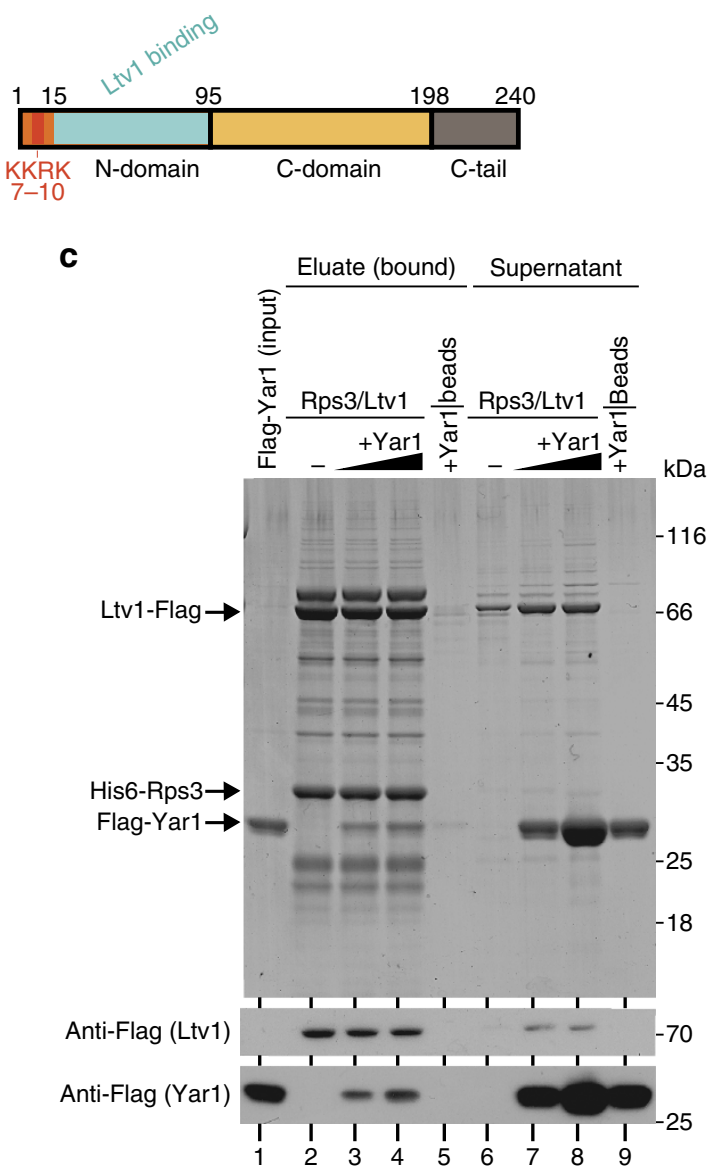

Figure 3 | Ltv1 releases Yar1 upon binding to the Rps3 N-domain. (a) Yar1 and Ltv1 have overlapping binding sites on the Rps3 N-domain. Different Rps3 truncations fused to the Gal4 DNA-binding domain (G4DBD) were tested for Y2H interaction with Yar1 (left panel), Enp1 or Ltv1 (right panel) fused to the Gal4 activation domain (G4AD). Growth on -His and -Ade plates, as well as white colony colour on -Leu-Trp plates, is indicative of a strong interaction. Both Yar1 and Ltv1 interact with N-terminal Rps3 fragments, whereas C-terminal Rps3 fragments do not mediate Y2H interaction with Yar1 or Ltv1. Below, the deduced binding domains, highlighted in blue colour, for each protein are illustrated schematically. (b) Ltv1 competes with Yar1 for Rps3 binding. Purified His6-Rps3/Yar1 complex immobilized on Ni-NTA agarose (and as control empty Ni-NTA agarose beads) was incubated either with buffer or with an $\sim 6$-fold molar excess (over Rps3) of purified Ltv1-Flag and/or Enp1-FPA (Flag-ProteinA). Supernatants of the incubation and eluates (containing His6-Rps3 and bound proteins) were analysed by SDS-PAGE and Coomassie staining or western blotting. (c) Yar1 competes only inefficiently with Ltv1 for Rps3 binding. Purified His6-Rps3/Ltv1-Flag complex immobilized on Ni-NTA agarose (and empty beads as negative control) was incubated either with buffer or with an $\sim 1.5$-fold (lanes 3 and 7) or 6-fold (lanes 4 and 8) molar excess of purified Flag-Yar1. Eluates containing His6-Rps3 and bound proteins and supernatants were analysed by SDS-PAGE and Coomassie staining or western blotting.

predominantly found in the soluble fraction (Fig. 4f). Noticeably, the assembly factors Rio2 and Tsr1 also accumulated on pre-ribosomes in the ltv1(S336A/S339A/S342A) mutant (Fig. 4f), supporting the idea that the phosphorylationdependent Ltv1 release is a prerequisite for downstream cytoplasmic maturation steps. In addition, likewise to an $l t v 1 \Delta$ strain, the mutant was inhibited in the final rRNA processing step, as revealed by the accumulation of immature $20 \mathrm{~S}$ pre-rRNA and the reduction of mature 18S rRNA levels (Fig. 4g). Moreover, also other ltv1 mutants harbouring serine 
a

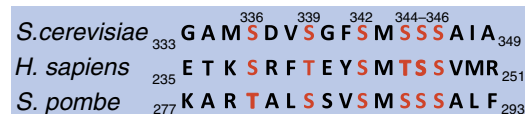

S. pombe ${ }_{277}$ K A R TA L S S VS M S S SA L F ${ }_{293}$

\section{d}

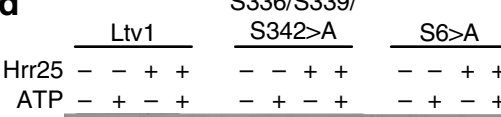

b

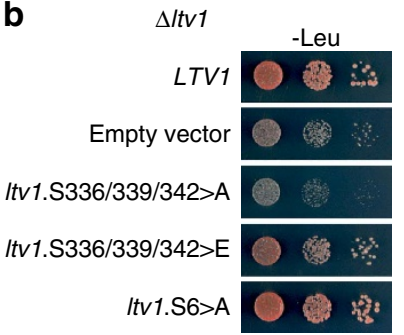

C

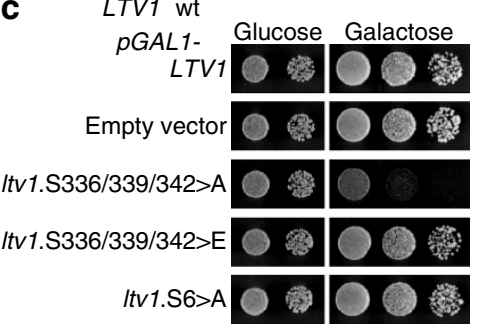

e

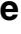

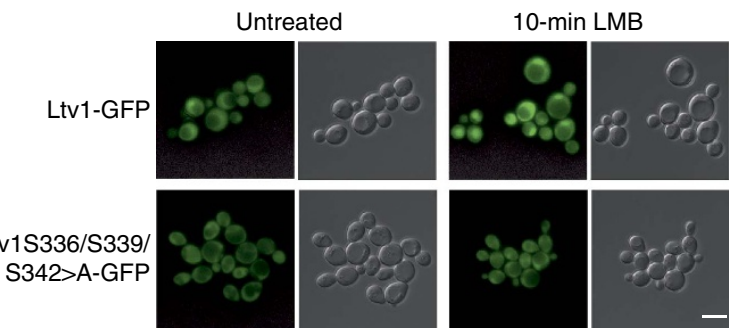

$\mathbf{f}$

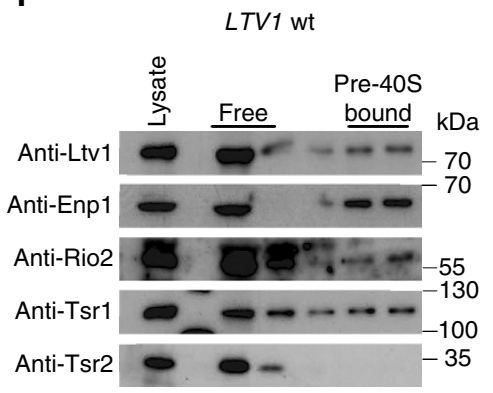

Itv1 $\mathrm{S} 336 / 339 / 342>A$

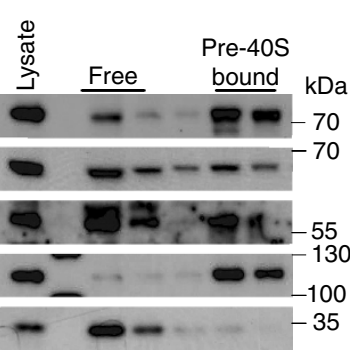

h

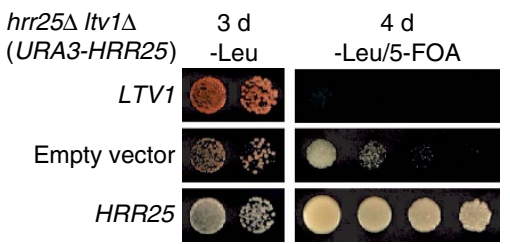

Itv1 S6>A
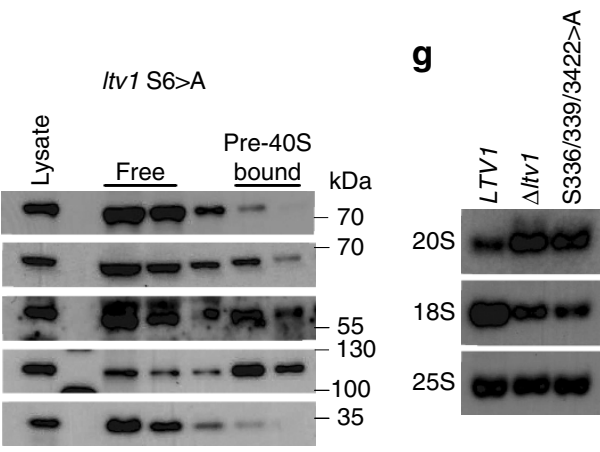

Figure 4 | Cytoplasmic Ltv1 release is triggered by phosphorylation of serines 336, 339 and 342, and is essential for cell growth. (a) Sequence alignment reveals a conserved serine/threonine-rich motif in Ltv1 as putative target for phosphorylation by Hrr25/CK1 / $/ \varepsilon$. (b) Growth phenotypes of the indicated Itv1 phosphomutant strains at $23^{\circ} \mathrm{C}$ (3-day (d) incubation). (c) Wild-type LTV1 and Itv1 phosphomutant alleles were overexpressed from the galactose inducible GAL1-10 promoter in an LTV1 wild-type background. Growth was examined after incubation on glucose (repressed) or galactose (induced) containing plates at $23^{\circ} \mathrm{C}$ for 3 and $7 \mathrm{~d}$, respectively. (d) The indicated Flag-tagged Ltv1 proteins were incubated with Hrr25 in the presence or absence $( \pm$ ) of ATP. Samples were analysed by SDS-PAGE and Coomassie staining (upper panel) or western blotting (lower two panels). Phosphorylated Ltv1 was detected with the phosphate-binding reagent Phos-tag and is also recognizable as a slower migrating band. (e) The localization of wild-type Ltv1GFP or Ltv1(S336A/S339A/S342A)-GFP was examined in LMB-sensitive crm1 mutant cells before and after 10 min of incubation with LMB. Note that due to the $\mathrm{crm} 1$ mutation, Ltv1-GFP, but not the phosphomutant, partially localizes in the nucleus already in untreated cells. After 10 min of treatment, a strong nuclear accumulation of Ltv1-GFP can be observed, while the phosphomutant protein is still localized in the cytoplasm. Scale bar, $5 \mu \mathrm{m}$. (f) Cell lysates of wild-type LTV1 or Itv1 phosphomutant strains were analysed by sucrose gradient centrifugation, and the upper fractions of the gradients containing soluble proteins (free) to $40 \mathrm{~S}$ subunits (pre-40S bound) were analysed by western blotting. Tsr2 was detected as marker for the soluble fraction. (g) Ltv1 phosphorylation is required for the final rRNA processing step. Indicated strains were analysed by northern blotting with probes directed against immature $20 S$ and mature $18 S$ and $25 S$ rRNA species. (h) Phosphorylation-dependent pre-40S release of Ltv1 is the essential cellular function of Hrr25. A hrr25 Itv1A strain with wild-type HRR25 on a URA3-plasmid was transformed with vector or plasmids carrying LTV1 or HRR25. The ability to lose the URA3-HRR25 plasmid was assessed on synthetic dextrose (SD) -leu plates containing 5-Fluoroorotic acid (5-FOA) incubated at $30^{\circ} \mathrm{C}$ for $4 \mathrm{~d}$. Although a hrr $25 \Delta$ strain is inviable when LTV1 is present, the strain is viable in the absence of LTV1.

to alanine exchanges, but not ltv1(S6A), displayed rRNA processing defects (Supplementary Fig. 5d).

The kinase Hrr25 is essential for yeast viability, and is involved in a wide range of cellular processes (see for example, refs 30-32).
Since we observed severe defects for the ltv1-phosphorylation mutants, we reasoned that the role of Hrr25 in Ltv1 release might be particularly important. Indeed, HRR25 became dispensable in an ltv1 deletion strain (Fig. 4h). While this manuscript was in 
preparation, similar effects were reported upon shutdown of Hrr25 expression in ltv 1 deletion strains ${ }^{7}$. Hence, Ltv1 phosphorylation and its subsequent pre-40S release is the essential cellular function of Hrr25.

40S maturation requires formation of Rps3 N-domain contacts. As long as Ltv1 is bound to $18 \mathrm{~S}$ rRNA helix 41, this part of the rRNA is not available for binding of the Rps $3 \mathrm{~N}$-domain via arginine 9 (R9) and lysine 8 (K8; Fig. 5a) ${ }^{21,24}$. To assess the physiological relevance of these Rps3-rRNA contacts, we analysed the phenotypes of mutants carrying exchanges within these residues. In addition, we mutated the adjacent lysine 7 (K7) and lysine 10 (K10), which engage in interaction with the r-protein Rps20 (Fig. 5a). Substitution of K8 and R9 (rRNA contacts) or K7 and $\mathrm{K} 10$ (Rps20 contacts) to alanine did not result in substantial growth defects, while combined substitution of all four residues to alanines (KKRK $>A$ ) resulted in significantly reduced cell growth (Fig. 5b). Remarkably, an even more severe growth defect was observed after mutating $\mathrm{K} 7$ and $\mathrm{K} 10$ to negatively charged glutamate and aspartate, respectively; thereby inducing a repulsion from the interacting negatively charged glutamate (E115) and aspartate (D113) residues of Rps20 (Fig. 5a,b). We have previously reported that the N-terminal KKRK-motif (amino acids 7-10) within Rps3 constitutes a functional nuclear localization signal (NLS) ${ }^{22}$. However, as fusion of the strong SV40-NLS to the rps3 mutant alleles did not rescue the growth defects, the observed growth phenotypes can be attributed to the loss of interactions with $18 \mathrm{~S}$ rRNA and Rps20, rather than to a nuclear import defect (Supplementary Fig. 6). Further analyses revealed that $r p s 3(\mathrm{KKRK}>\mathrm{A})$ and $r p s 3(\mathrm{~K} 7 \mathrm{E} / \mathrm{K} 10 \mathrm{D})$ strains displayed severe rRNA processing defects, as evidenced by the accumulation of unprocessed 20S pre-rRNA and the decrease of mature $18 \mathrm{~S}$ rRNA levels (Fig. 5c). Hence, the formation of the ionic contacts between Rps3 and Rps20, as well as between Rps3 and rRNA, is not only necessary for the integrity of mature $40 \mathrm{~S}$ subunits, but appears to be also required for $40 \mathrm{~S}$ subunit maturation.

Rps3 N-domain assembly releases Ltv1 from pre-40S particles. Notably, we observed a synthetically enhanced growth defect of the $r p s 3(\mathrm{~K} 7 \mathrm{~A} / \mathrm{K} 10 \mathrm{~A})$ mutant with ltv1-phosphorylation mutants, raising the idea that, in addition to Ltv1 phosphorylation, also the final assembly of the Rps3 $\mathrm{N}$-domain might be involved in releasing Ltv1 from pre-ribosomal particles (Fig. 5d). To address the role of Rps3 residues K8, R9, K7 and K10 in Ltv1 release, we made use of a GFP fusion reporter construct containing a deletion of the recently reported C-terminal nuclear export sequence (NES) of Ltv1 (ref. 27). In contrast to the localization of wild-type Ltv1-GFP, Ltv1 $\triangle$ NES-GFP strictly localized to the nucleus of RPS3 wild-type cells (Fig. 5e). Strikingly, the localization shifted to the cytoplasm when monitored in the rps 3 assembly mutants, hence revealing a defect in pre-40S release of Ltvl (Fig. 5e). While $r p s 3(\mathrm{~K} 7 \mathrm{~A} / \mathrm{K} 10 \mathrm{~A})$ and $r p s 3(\mathrm{~K} 8 \mathrm{~A} / \mathrm{R} 9 \mathrm{~A})$ mutants showed a partial mislocalization of the Ltv1 $\Delta$ NES-GFP reporter, the localization was completely shifted to the cytoplasm in the rps3(KKRK $>\mathrm{A}$ ) and $r p s 3(\mathrm{~K} 7 \mathrm{E} / \mathrm{K} 10 \mathrm{D})$ mutants. Furthermore, these $r p s 3$ mutants showed increased amounts of Ltv1 in the $40 \mathrm{~S}$ fraction after sucrose gradient centrifugation (Fig. 5f). Remarkably, the assembly factors Enp1, Rio2 and Tsr1 also accumulated on pre$40 \mathrm{~S}$ particles (Fig. 5f). We conclude that the residues of the Rps3 $\mathrm{N}$-domain involved in interaction with $18 \mathrm{~S}$ rRNA $\mathrm{H} 41$ and with Rps20 are required for efficient Ltv1 release from pre-40S particles, thereby permitting progression of cytoplasmic $40 \mathrm{~S}$ maturation.

If the assembly defects displayed by the rps3(K7E/K10D) mutant were due to the inability in forming the ionic contacts with Rps20, similar phenotypes should be observed when the contacts are disrupted by mutating RPS20. Indeed, an rps20(D113K/E115K) mutant displayed a slow-growth phenotype and an Ltv1 release defect (Fig. 6a,e,f). Furthermore, the rps20 mutant was genetically linked to $l t v 1$-phosphorylation mutants (Fig. 6d) and showed a $20 \mathrm{~S}$ pre-rRNA processing defect (Fig. 6c). Strikingly, when the contact between Rps3 and Rps20 was restored by simultaneously mutating both interaction sites $(r p s 3(\mathrm{~K} 7 \mathrm{E} / \mathrm{K} 10 \mathrm{D}) / r p s 20(\mathrm{D} 113 \mathrm{~K} / \mathrm{E} 115 \mathrm{~K}))$, thus reverting the charge repulsion, cells almost grew at wild-type rates again (Fig. 6b) and the rRNA processing defect was partially rescued (Fig. 6c). Notably, as this rps3-rps20 double mutation restores the Rps3-Rps20 interaction while leaving the Rps3-NLS non-functional, the normal growth suggests that apart from the import route involving this $\mathrm{N}$-terminal NLS, also additional, redundant Rps3 import pathways have to exist. We conclude that the phenotypes observed in rps3 and rps20 mutants can be attributed to the inability to form the contact between Rps3 and Rps20. This interaction is necessary for Ltv1 release, but is likely also important for the function of the mature $40 \mathrm{~S}$ subunit.

Ltv1 is positioned between the Rps3 N-domain and Rps20. On the basis of our results linking the cytoplasmic Ltv1 release to the association of the Rps3 N-domain with Rps20, and considering that the Ltv1-rRNA crosslinking site in helix 41 is physically close to Rps20, we reasoned that in pre-40S particles Ltv1 might be in direct contact not only with Rps3 but also with Rps20. Indeed, following a two-step affinity purification, we detected a robust in vitro interaction between Ltv1 and Rps20 (Fig. 7a, left panel). We hypothesized that Ltv1 is clamped between Rps3 and Rps20 on pre-40S ribosomal subunits, thereby preventing an interaction between the two r-proteins at a too early maturation stage. To determine the orientation of Ltv1 on pre-40S particles, we mapped the Rps3 and Rps20 interaction sites of Ltv1. Consistent with a previous study ${ }^{27}$, we found a fragment close to the Ltv1 $\mathrm{N}$ terminus (amino acids 57-105) to mediate the $\mathrm{Y} 2 \mathrm{H}$ interaction with the Rps3 $\mathrm{N}$-domain (Fig. $7 \mathrm{~b}, \mathrm{c}$ ). In contrast, the in vitro interaction with Rps20 is mediated by the C-terminal part of Ltv1, with amino acids 310-463 being sufficient for interaction with Rps20 (right panel in Fig. 7a and Fig. 7c). Hence, considering the proposed elongated structure of Ltv1, spanning from rRNA helix 16 to helix 41 , there has to be a significant distance between the Rps3 N-domain and Rps20 in pre-40S particles.

To predict the position of the Rps3 $\mathrm{N}$-domain in pre-40S particles, we considered the possibility that Rps3 or the Rps3/Yar1 complex is incorporated into early ribosomal precursors with the rotated domain arrangement that we observed in the Rps3/Yar1 solution structure. We modelled the Rps $3 / Y a r 1$ complex both into the $40 \mathrm{~S}$ crystal structure and into the pre-40S cryo-electron microscopy structure by assuming the same orientation of the Rps3 C-domain as in mature $40 \mathrm{~S}$ subunits (Fig. 7d; Supplementary Fig. 7). Indeed, the Rps3 N-domain protruded away from the $40 \mathrm{~S}$ surface in these models, preventing it from interaction with rRNA and Rps20. This conformation might allow Yar1 to remain bound to the Rps3 $\mathrm{N}$-domain until being removed by Ltv1. Moreover, this arrangement would also leave sufficient space for binding of Ltvl to its interaction site at rRNA helix 41 , and would orient the Rps3 $\mathrm{N}$-domain towards rRNA helix 16, constituting the second Ltv1 crosslinking site $\mathrm{e}^{21}$.

We conclude that while the Ltv1 N-domain, bound to the Rps3 $\mathrm{N}$-domain, is likely positioned close to helix 16 , the C-terminal part of Ltv1 (which also contains the Hrr25 phosphorylation site) interacts with Rps20 and is oriented towards helix 41 (Fig. 7c,d). 
a

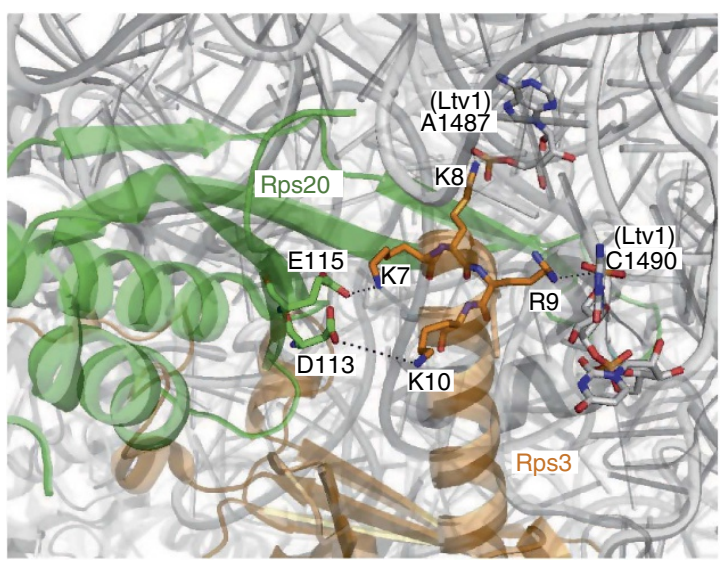

b

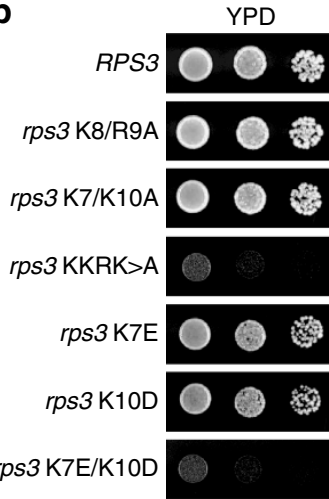

d
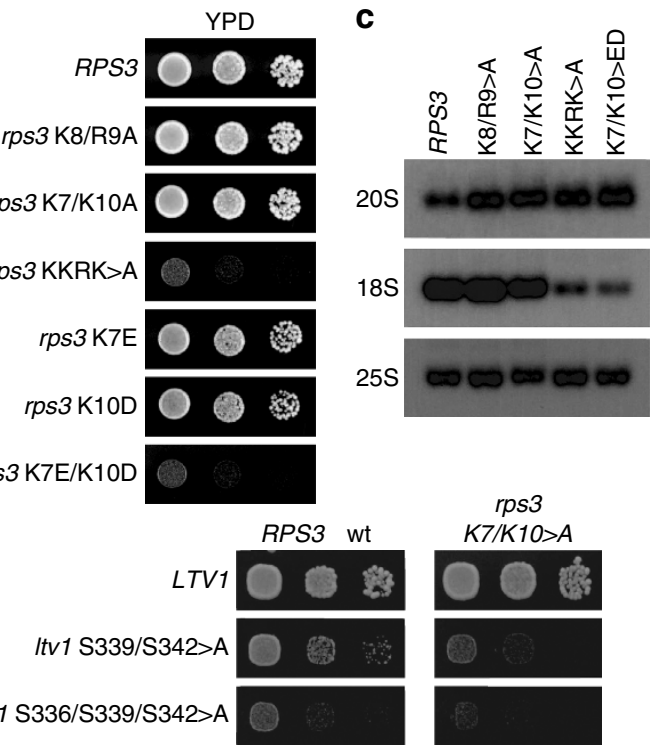

e
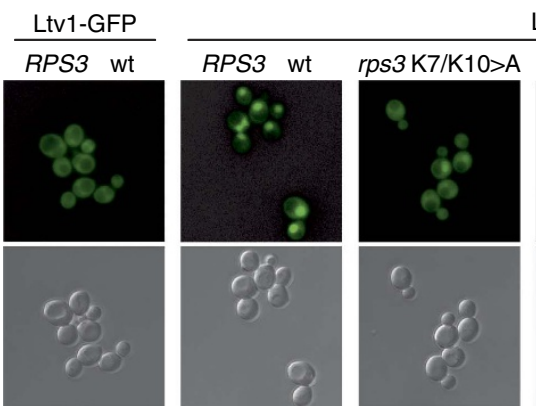

Ltv1 $\triangle$ NES-GFP

-Leu-Trp
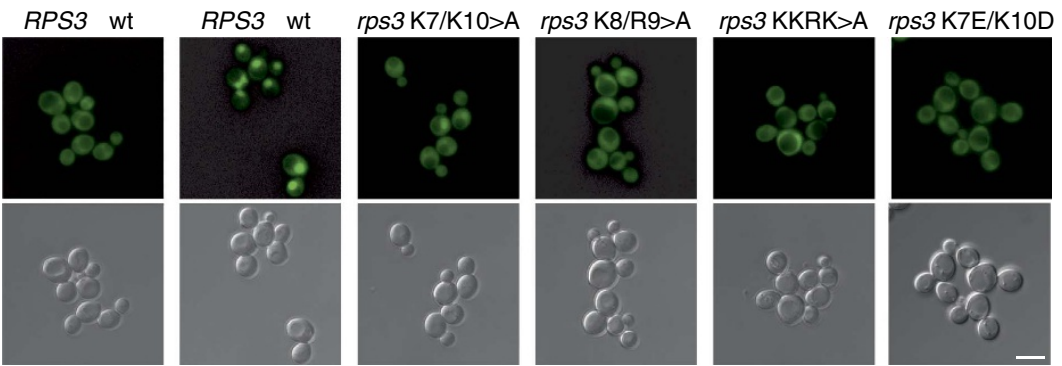

f
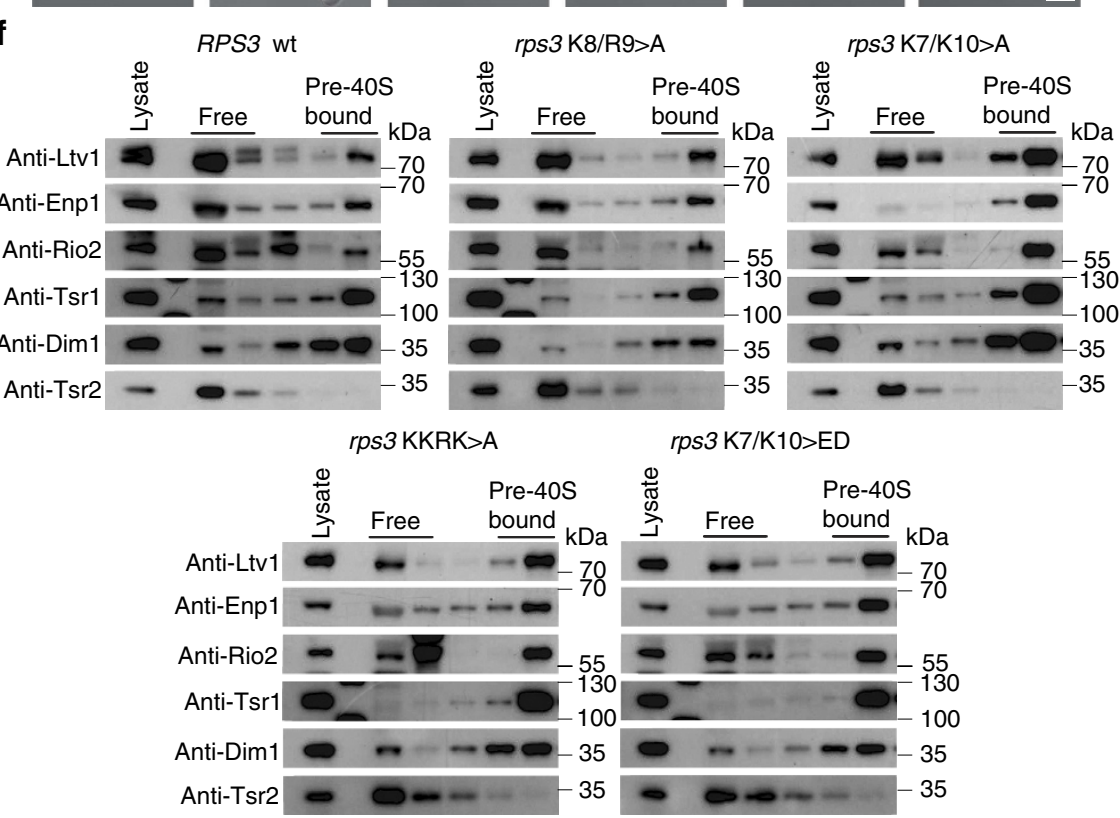

Figure 5 | Assembly of the Rps3 $\mathbf{N}$-domain releases Ltv1 from pre-40S particles and is required for final $40 \mathrm{~S}$ maturation steps. (a) N-terminal Rps3 residues form ionic contacts with negatively charged Rps20 residues and with rRNA nucleotides A1487 and C1490 around the rRNA-binding site of Ltv1 in helix 41 (PDB 3U5B and 3U5C). (b) Residues of the Rps3 N-domain interacting with Rps20 and rRNA are required for optimal cell growth. Growth phenotypes of the indicated rps 3 alleles harbouring amino acid substitutions within the $\mathrm{N}$-terminal residues $\mathrm{K} 7, \mathrm{~K} 8, \mathrm{R} 9$ and $\mathrm{K} 10$ were examined after incubation at $23^{\circ} \mathrm{C}$ for 3 days (d). (c) N-terminal rps3 mutants display $20 \mathrm{~S}$ pre-rRNA processing defects. The amounts of immature $20 \mathrm{~S}$ and mature $18 \mathrm{~S}$ rRNA in the indicated rps 3 mutants were analysed by northern blotting. (d) rps3(K7A/K10A) has a synthetically enhanced growth phenotype with Itv1-phosphorylation mutants. The genetic interaction between the indicated alleles was examined after incubation at $23^{\circ} \mathrm{C}$ for $4 \mathrm{~d}$. (e) The localization of Ltv1 $\Delta N E S$-GFP was examined in cells expressing the indicated rps3 alleles. Note that, in contrast to wild-type Ltv1-GFP, Ltv1 1 NES-GFP displays a strictly nuclear localization in RPS3 cells that is shifted to the cytoplasm in the rps3 mutants. Scale bar, $5 \mu \mathrm{m}$. (f) rps3 mutants accumulate Ltv1 and further cytoplasmic assembly factors on pre-40S particles. Lysates of the indicated rps 3 mutants were analysed by $7-45 \%$ sucrose gradient centrifugation, and the upper fractions of the gradients containing soluble proteins (free) to $40 \mathrm{~S}$ subunits (pre-40S bound) were analysed by western blotting with the indicated antibodies. 
a

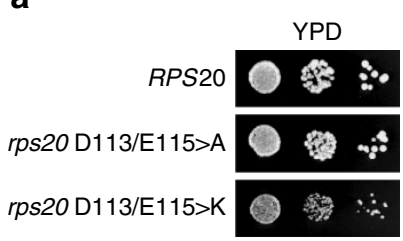

b

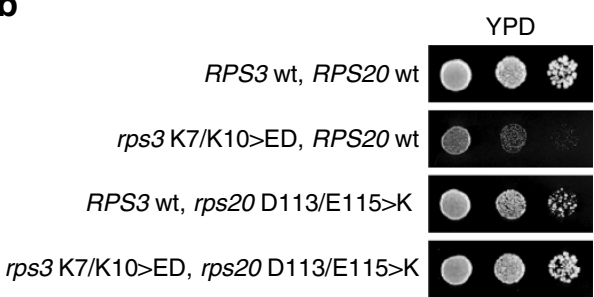

d

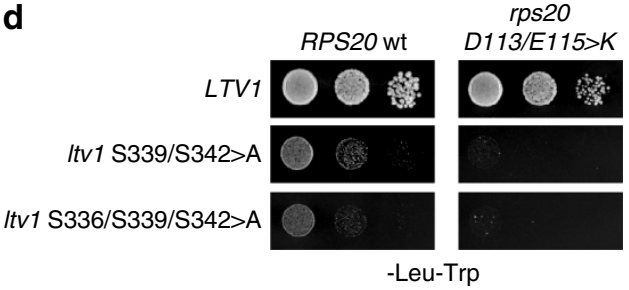

e
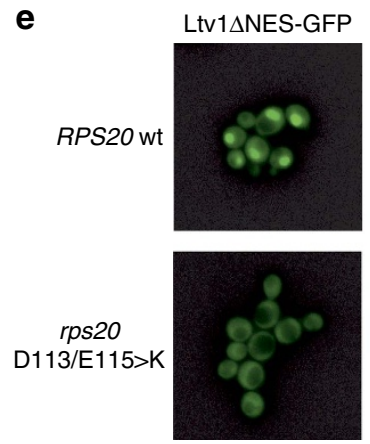

f

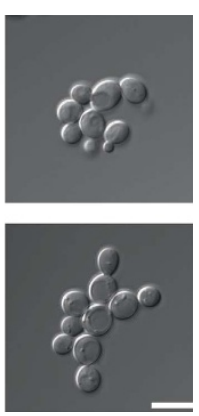

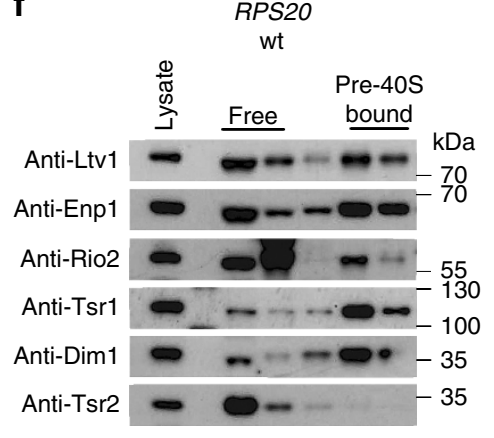

c

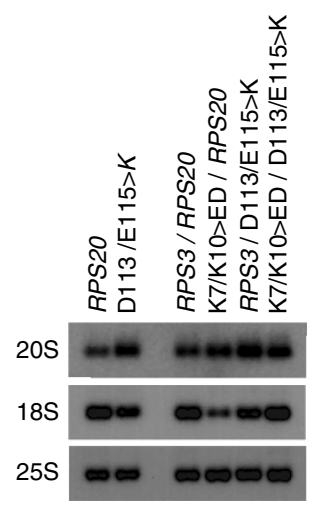

Figure 6 | Cytoplasmic Ltv1 release is inhibited in the rps20(D113K/E115K) mutant. (a) Growth phenotypes of wild-type RPS20 and rps20(D113K/E115K) mutant after incubation at $23^{\circ} \mathrm{C}$ for 3 days (d). (b) Reversion of the charge repulsion of $r p s 3 / r p s 20$ mutants restores cell growth to wild-type rates. The growth phenotypes of the indicated mutants were assessed after incubation at $23^{\circ} \mathrm{C}$ for $3 \mathrm{~d}$. (c) The $20 \mathrm{~S}$ pre-rRNA processing defect of rps3(K7E/K10D) and $r p s 20(D 113 K / E 115 K)$ single mutants is partially rescued in the $r p s 3 / r p s 20$ double mutant. The indicated rps 3 and rps 20 mutants were analysed by northern blotting. (d) Synthetically enhanced growth phenotype of rps20(D113K/E115K) with Itv1 phosphomutants. The genetic interaction between the indicated alleles was assessed after incubation at $23^{\circ} \mathrm{C}$ for $4 \mathrm{~d}$. (e) Ltv1 1 NES-GFP mislocalizes to the cytoplasm in rps20(D113K/E115K) cells. The localization of Ltv1ANES-GFP was examined in RPS20 and rps20(D113K/E115K) cells. Scale bar, $5 \mu \mathrm{m}$. (f) Lysates of RPS20 and rps20(D113K/E115K) cells were analysed by $7-45 \%$ sucrose gradient centrifugation, and the upper fractions of the gradients containing soluble proteins (free) to $40 S$ subunits (pre405 bound) were analysed by western blotting with the indicated antibodies.

\section{Discussion}

In this study, we have uncovered an intriguing mechanism involving a peculiar dimeric and rotated structure of the r-protein Rps3, and provided functional insights into the events underlying the stable integration of Rps3 during the final structural reorganization within the $40 \mathrm{~S}$ beak region. On the basis of our data, we suggest a model for the assembly path of Rps3, which is shown in Fig. 8 and discussed below.

Newly synthesized Rps3 is captured co-translationally by its chaperone Yar1 (ref. 33), which protects the N-terminal part of Rps3. Following Rps3 synthesis, its C-domain dimerizes with a second Rps3 molecule by domain swapping. Our in vitro data suggest that the structural properties of the complex allow binding of two Yar1 molecules (one to each Rps3 N-domain); however, in vivo, only one Yarl is present in this complex. This suggests that in vivo a mechanism exists that either prevents a second Yar1 from binding or that removes one Yarl molecule quickly after formation of the tetrameric Rps3/Yar1 complex. Considering that Rps3 harbours an NLS in its N-domain (KKRK-motif, amino acids 7-10 (ref. 22)) directly adjacent to the Yarl-binding site (amino acids 11-30; Fig. 3a, left panel), it is unlikely that Yar1 and an importin can bind this region at the same time. It is tempting to speculate that importin binding may release one Yar1 molecule from the Rps3 dimer.
Another function of Rps3 dimerization might be protection from aggregation. Considering that Yar1 only protects the Rps3 $\mathrm{N}$-domain but not the $\mathrm{C}$-domain, which comprises the main rRNA-binding surface, dimerization likely serves to shield this part of the protein from unspecific interactions. In addition, the dimeric conformation might be cost-effective as it allows simultaneous targeting of two Rps3 molecules to ribosome assembly. Finally, the conformation of the Rps3/Rps3/Yar1 complex might be required to guide the incorporation of rotated Rps3 into pre-ribosomal particles.

Dissociation of the Rps3/Rps3/Yar1 complex occurs upon nuclear assembly of Rps3 into pre-ribosomal particles. Since domain swapping masks the major rRNA-binding site of the Rps3 C-domain, Rps3 dimers are presumably separated by competition with rRNA. It is not clear yet if a distinction is made whether the Yar1-bound Rps3 or the free Rps3 is incorporated. In our view, both events are possible-binding of Yar1-bound Rps3 would result in the subsequent release of Yar1 upon Ltv1 binding, while binding of free Rps3 would allow the direct binding of Ltv1. Once bound, Ltv1 prevents the final, salt-stable incorporation of the Rps3 N-domain in two ways: (1) the C-terminal part of Ltv1 interacts with Rps20 and the adjacent rRNA helix 41, hence occupying the final binding site of the Rps3 N-domain; and (2) the N-terminal part of Ltv1 binds to the Rps3 
$\mathrm{N}$-domain in proximity to rRNA helix 16 , thereby fixing the Rps3 $\mathrm{N}$-domain in its rotated conformation. We postulate that, in the cytoplasm, Ltv1 release is accomplished by a two-step mechanism: Hrr25-dependent phosphorylation of Ltv1 at serines 336, 339 and 342 leads to an electrostatic repulsion of the C-terminal part of Ltv1 from its rRNA-binding site at helix 41 and/or from Rps20, thereby making the site available for Rps3 binding. This event would loosen the 'Ltv1 clamp' and relax the fixed conformation of Rps3, thus allowing a rotational movement of the Rps3 N-domain into its final assembly position where it interacts with Rps20 and rRNA helix 41. The force generated during the flip of the Rps3 $\mathrm{N}$-domain may finally pull the Rps3-bound N-terminal part of Ltv1 off the second rRNA-binding site at helix 16, thereby leading to Ltv1 release. a

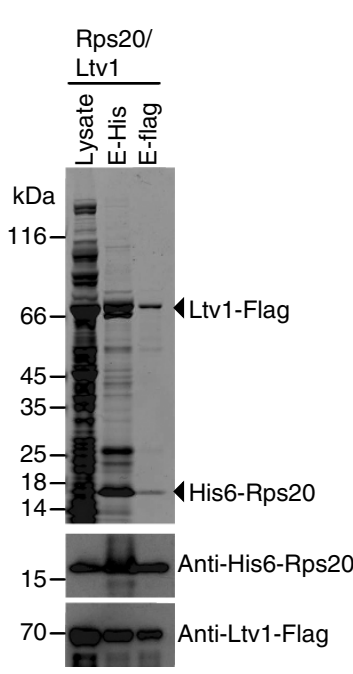

d

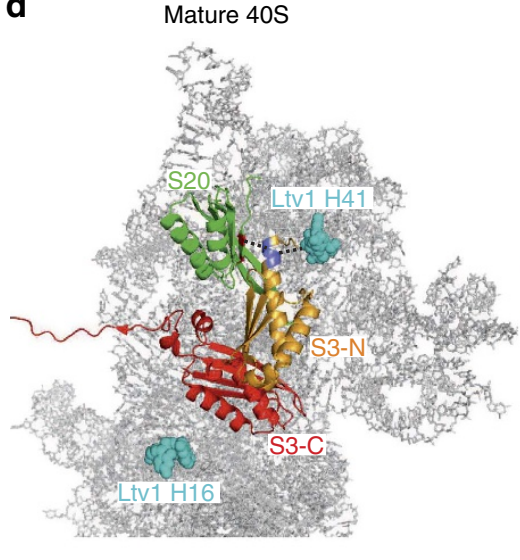

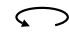

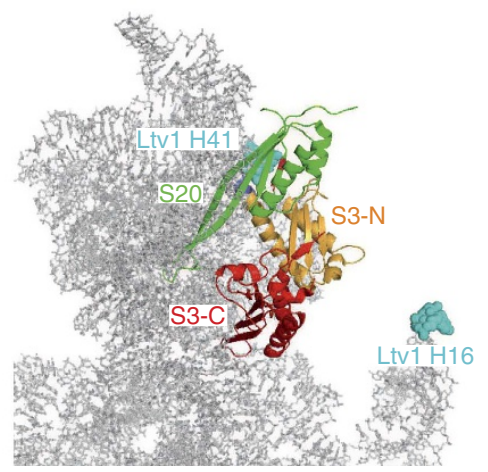

Rps20/Ltv1-

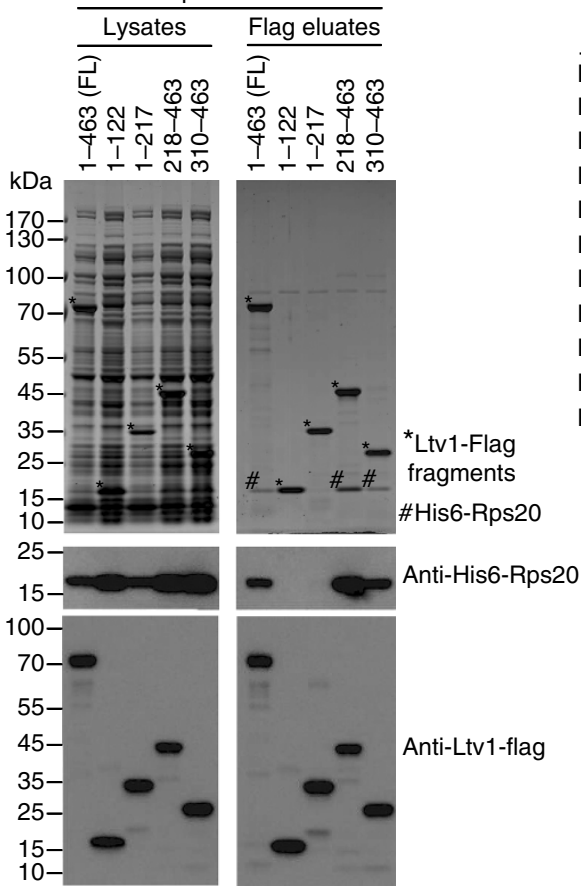

40S with rotated Rps3
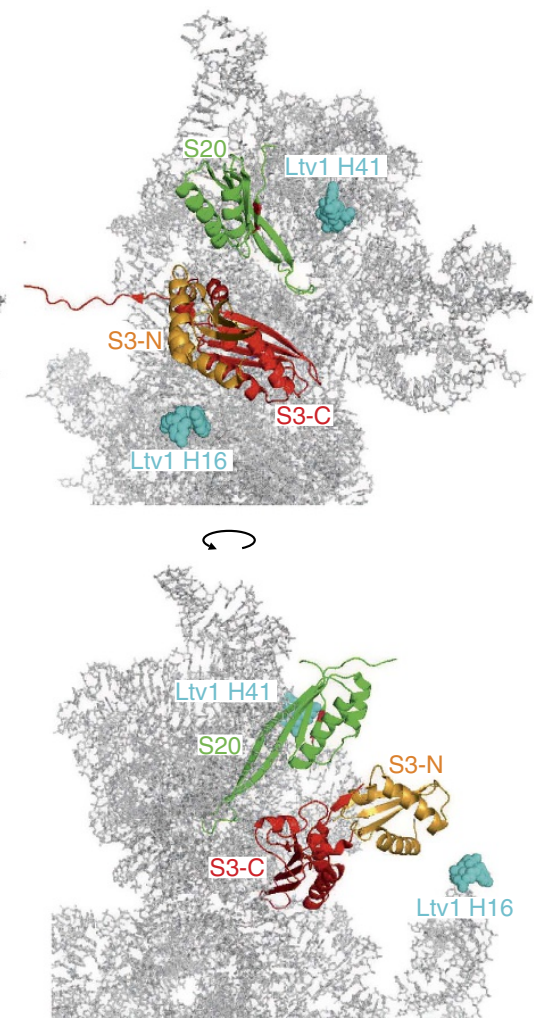

b

\begin{tabular}{|c|c|c|c|c|}
\hline G4DBD & G4AD & & $-H i s$ & -Ade \\
\hline $\operatorname{Rps} 3(11-95)$ & Ltv1 & & 0009 & $000 \%$ \\
\hline $\operatorname{Rps} 3(1$ & $\operatorname{Ltv} 1(57-463)$ & 000 : & 000 紜 & 000 管 \\
\hline$p s 3(1$ & Ltv1(120-46 & & & \\
\hline $\mathrm{ss} 3$ & $\operatorname{Ltv} 1(2$ & 0 \& & & \\
\hline os3 & Ltv1(1-219) & 000 & O०० क & $000=$ \\
\hline os31 & Ltv1(1 & 0000 & 000 . & 0008 \\
\hline ss 3 & & 000 \% & 1000 . & $000 \mathrm{E}$ \\
\hline ps3(1 & Ltv1(57-219) & $000 \%$ & 000 क & 000 늉 \\
\hline $\operatorname{Rps} 3(1$ & Ltv1(57-122) & $000 \%$ & 000 & 00.9 \\
\hline ps3(11-95) & Ltv1 $157-1$ & 000 & $000 ;$ & $00=9$ \\
\hline ps3(11-9 & & & & \\
\hline
\end{tabular}

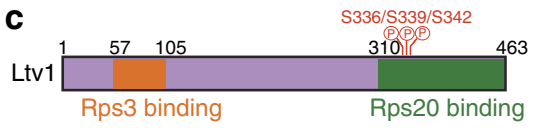

40S with Rps3/Yar1 complex
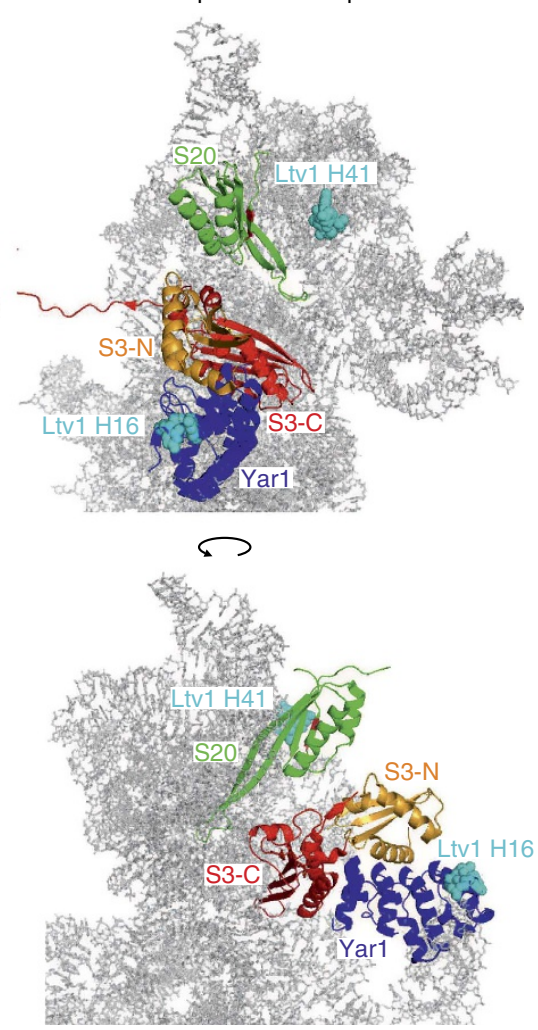
Several bacterial r-proteins form their interactions with different rRNA-binding sites in sequential stages rather than in one step ${ }^{34}$. An example is r-protein S4, which initially only binds to part of its rRNA-binding site and induces folding events in the rRNA, which then results in the formation of additional interactions with the rRNA ${ }^{35}$.

It was observed that association of several eukaryotic r-proteins, including Rps3, with early pre-ribosomal particles is sensitive to salt treatment, and that salt stability of binding of these proteins increases with the progression of ribosome assembly $8,25,36$. Consequently, it was proposed that also eukaryotic r-protein assembly occurs stepwise, with only weak initial association to rRNA that is strengthened upon progression of ribosome maturation; however, it was not clear how such a transition might be achieved ${ }^{8,25,36}$. This study provides mechanistic insights into how an r-protein, which is initially attached loosely via only one domain, reaches its mature and stable conformation upon incorporation of the second interaction domain. Moreover, the direct coupling of r-protein assembly with the release of an assembly factor might represent a proofreading mechanism ensuring that only properly assembled $40 \mathrm{~S}$ precursors can undergo final maturation steps and gain access to the pool of translationally active subunits. Another r-protein, Asc1, was reported to form dimers while exerting its extra-ribosomal function in cell signalling ${ }^{37}$, and it remains open whether Asc1 dimerization also precedes its ribosome incorporation. Future studies will have to determine whether other r-proteins are also assembled by a stepwise integration of individual domains and whether dimerization, as observed here for Rps3, is also used in their assembly paths.

\section{Methods}

Strains and plasmids. Saccharomyces cerevisiae strains used in this study are listed in Supplementary Table 2, and were obtained by established recombination, mating and tetrad dissection procedures. Plasmids used in this study were created using standard recombinant DNA techniques and are listed in Supplementary Tables 3 and 4 .

SAXS data collection, analyses and modelling. SAXS data with direct injection of the sample were collected on beamline BM29 at European Synchrotron Radiation Facility (ESRF; Grenoble, France) and SAXS data with size exclusion separation before data collection ${ }^{38}$ were collected on beamline SWING at the SOLEIL synchrotron (Saint-Aubin, France).

For direct injection, protein samples were centrifuged at $13,200 \mathrm{~g}$ for $10 \mathrm{~min}$ before data acquisition and concentration was measured with NanoDrop spectrophotometer (Thermo). The samples were then loaded at a flow rate of $200 \mu \mathrm{min}^{-1}$ in the SAXS capillary flow cell. Experiments on BM29 were done at an electron energy of $8 \mathrm{keV}$, and data were recorded on a Pilatus $1 \mathrm{M}$ detector (Dectris). Ten 1-s exposure frames were collected for each sample. For size exclusion chromatography, samples were loaded on an Agilent SEC-3 size-exclusion chromatography column connected to an Agilent high-performance liquid chromatography system at a flow rate of $200 \mu \mathrm{lmin}^{-1}$. Experiments on SWING were done at an electron energy of $13.32 \mathrm{keV}$, and data were recorded on

PCCD170 detector (AVIEX). One hundred frames, with exposure times of $1 \mathrm{~s}$ per frame, corresponding to the elution peak were collected at $288 \mathrm{~K}$ in the flow cell connected to high-performance liquid chromatography system. The same buffer was used for all data collection (Tris- $\mathrm{HCl} 50 \mathrm{mM}$ and $\mathrm{NaCl} 200 \mathrm{mM}, \mathrm{pH} 7.5$ ). Buffer scattering was collected in the same conditions or using the gel filtration profile before the void volume.

Data reduction, image conversion to $1 \mathrm{D}$ profile, absorption correction, scaling to absolute scale, buffer subtraction, averaging and peak analysis were carried out using the pipeline available on BM29 beamline or using the program FOXTROT available on the SWING beamline. Further processing and data analysis was done using the programs of the ATSAS suite ${ }^{39}$. Guinier plot analysis was performed using PRIMUS with scattering data at low-angle regions with $\mathrm{qmax} \times \mathrm{Rg}<1.3$. Pair distribution function was calculated with GNOM. Ab initio analysis was done with DAMMIF. In total, 50 DAMMIF calculations were averaged with DAMAVER to produce averaged and filtered shape. Homology models were made with MODELLER ${ }^{40}$

Modelling with SAXS data was done with DADIMODO ${ }^{41}$ using the core structural domain from the crystal structure and fitting for flexible $\mathrm{N}$ - and C-terminal extensions. The N-terminal (1-29) and C-terminal (207-214) extensions of Yar1 (1-214 including tag) were made flexible. For Rps3 (1-249 including tag), N-terminal (1-16) and C-terminal (203-249) extensions were modelled flexible. Flexibility between the N- and C- domains (101-105) was introduced.

Analysis of the fit between model and experimental data was done with CRYSOL.

The reduced chi-square calculation for a data set containing $N$ data points is defined as

$$
\chi^{2}=\frac{1}{N} \sum_{i=1}^{N} \frac{\left[I^{\mathrm{calc}}\left(q_{i}\right)-I^{\mathrm{obs}}\left(q_{i}\right)\right]^{2}}{\sigma_{i}^{2}}
$$

The relative residual is given by

$$
R(i)=\frac{I^{\text {calc }}\left(q_{i}\right)-I^{\text {obs }}\left(q_{i}\right)}{\sigma_{i}}
$$

For free chi-square calculation ${ }^{42}$, the data set is divided in $n_{S}$ bins, where $n_{S}$ is the number of Shannon channels.

$$
n_{S}=\frac{q_{\max } D_{\max }}{\pi}
$$

During a selection round $k$, a single data point is randomly chosen in each bin. $\chi^{2}$ is calculated with these $n_{S}$ points.

$$
\chi^{2}=\frac{1}{n_{S}} \sum_{i=1}^{n_{S}} \frac{\left[I^{\text {calc }}\left(q_{i}\right)-I^{\text {obs }}\left(q_{i}\right)\right]^{2}}{\sigma_{i}^{2}}
$$

$\chi^{2}$ free is the median of the $\chi^{2}$ calculated over $k$ rounds of selection. Typically, $k=1,001$.

The cumulative chi-square is given by

$$
\chi^{2}\left(q_{k}\right)=\frac{1}{k} \sum_{i=1}^{k} \frac{\left[I^{\mathrm{calc}}\left(q_{i}\right)-I^{\mathrm{obs}}\left(q_{i}\right)\right]^{2}}{\sigma_{i}^{2}}
$$

For chi-square calculation by momentum transfer bins, the data set is divided in $n_{S}$ bins.

For a bin $k\left(k\right.$ from 1 to $\left.n_{S}\right)$

$$
\chi^{2}(k)=\frac{n_{S}}{N} \sum_{i} \frac{\left[I^{\text {calc }}\left(q_{i}\right)-I^{\mathrm{obs}}\left(q_{i}\right)\right]^{2}}{\sigma_{i}^{2}}
$$

with $\frac{N(k-1)}{n_{S}}<i \leq \frac{N(k)}{n_{S}}$ used:

To model the full-length Rps/Yarl complex, the following stepwise strategy was

Figure 7 | Ltv1 is clamped between Rps20 and the rotated Rps3 N-domain. (a) The C-terminal part of Ltv1 binds Rps20. Left panel: His6-Rps20 and Ltv1Flag were co-expressed in E. coli. In a first purification step, His6-Rps20 was pulled down with Ni-NTA agarose and the obtained eluate was subjected to a second purification step with anti-Flag agarose. Cell lysate, His and Flag eluates were analysed by SDS-PAGE and Coomassie staining or western blotting. Right panel: after co-expression of His6-Rps20 with the indicated Flag-tagged $\mathrm{N}$ - and C- terminal Ltv1 truncations in E. coli, cell lysates were subjected to affinity purification with anti-Flag agarose. Cell lysates and Flag eluates were analysed by SDS-PAGE and Coomassie staining or western blotting. (b) N-terminal Ltv1 fragments interact with Rps3. Y2H domain mapping of Ltv1 truncations fused to the G4DBD and Rps3 fused to the G4AD reveal amino acids 57-105 as the Ltv1 interaction surface for Rps3 binding. (c) Rps3 and Rps20 interaction regions of Ltv1. Phosphorylated Ltv1 serines are indicated. (d) Initial incorporation of Rps3 in its rotated conformation allows pre-ribosomal assembly of Ltv1. Rps3 protein from the Rps3/Yar1 crystal structure (PDB 4BSZ, Fig. 1d, left panel) was modelled into the 40S crystal structure (PDB 3U5B and 3U5C) by superposition of the Rps3 C-domains. Compared to the position of the Rps3 N-domain within the mature ribosome (left panels), the Rps3 N-domain in the modelled conformation protrudes away from the $40 \mathrm{~S}$ surface (middle panels) and is accessible for interaction with Yar1 (right panel) or other assembly factors. This $\mathrm{N}$-domain rotation is compatible with Ltv1 binding at its rRNA-binding site at helix 41, which is occupied by the N-terminal Rps3 $\alpha$-helix in the mature 405 subunit. In contrast, the positioning of Yar1 is incompatible with Ltv1 binding to helix 16, further suggesting Yar1 release upon Ltv1 binding. The Rps3 N- and C-domains are shown in orange and red, respectively, the Ltv1-rRNA crosslink sites at helix 16 and helix 41 are shown as spheres coloured in cyan, Rps20 is displayed in green and Yar1 in blue. 


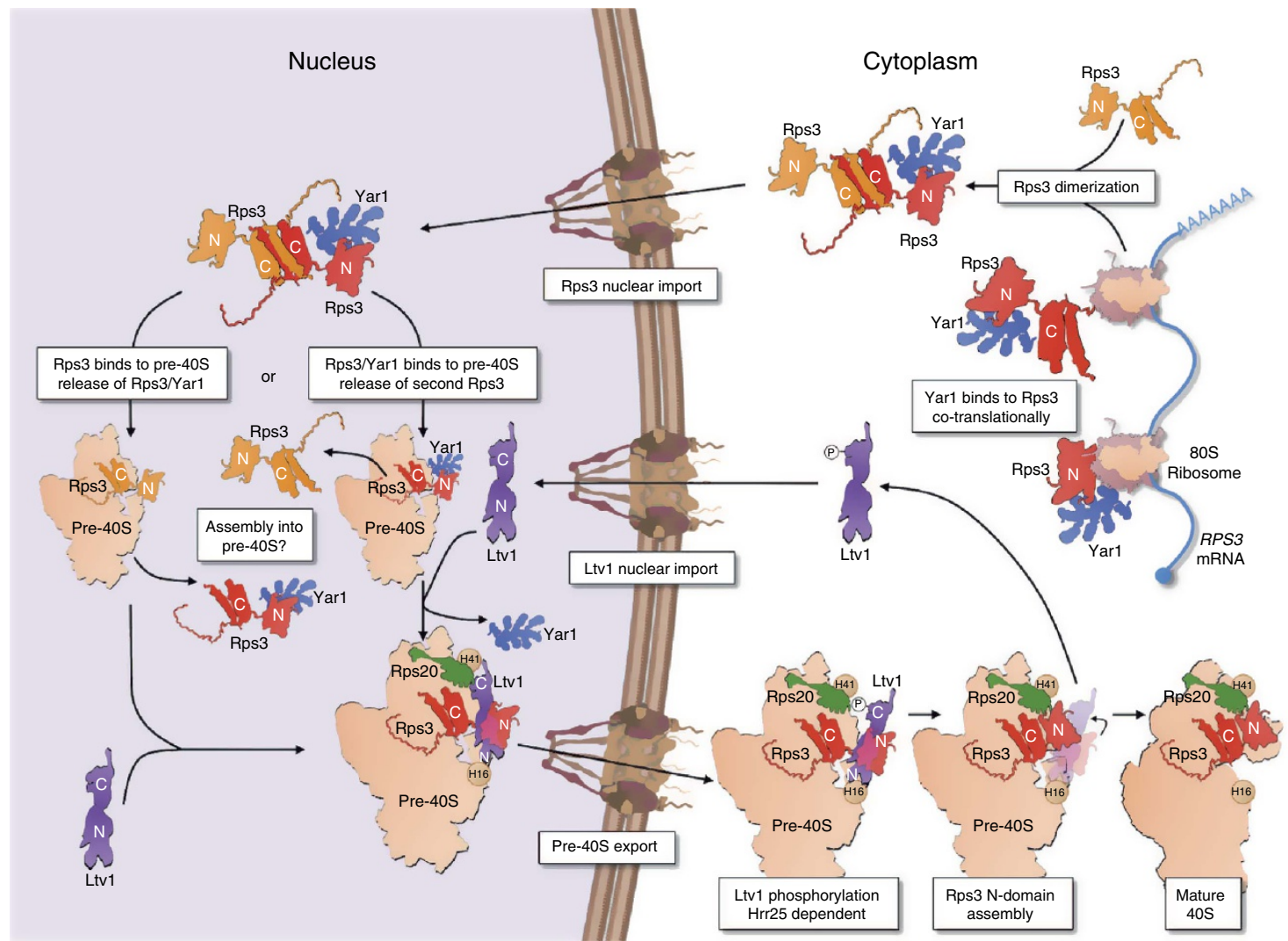

Figure 8 | Model of the assembly path of Rps3. See Discussion for details. Note that the released, second Yar1-bound or free Rps3 may be incorporated into another pre-40S subunit (not depicted).

We first used the SAXS data for Yarl full-length protein (Yar1-FL) to model Yarl alone. Model structures were generated with a rigid structural core corresponding to the residues observed in the crystal structure of the Yar1-Rps3 complex (Yar1(7-156)), and flexible N- and C-terminal extensions (29 N-terminal residues including 14 residues from the His-tag and $55 \mathrm{C}$-terminal residues). The conformations of the flexible extensions were optimized for a best fit against the experimental data, and were fitted into the independently determined molecular envelope. The models showed a good agreement with the experimental data with an overall $\chi^{2}$ of $1.06\left(\chi=1.03, \chi^{2}\right.$ free $\left.=1.04\right)$.

We next used SAXS data for the complex between the Yarl structural core lacking the $\mathrm{N}$ - and C-terminal extensions (Yar1(8-153)) and the Rps3 N-domain. The model was created by using the same conformations for the Yarl core and the Rps3 $\mathrm{N}$-domain as in the crystal structure. The $\mathrm{N}$ - and $\mathrm{C}$-terminal residues of each construct that are missing in the crystal structure were built and refined against the experimental data. This includes the $14 \mathrm{~N}$-terminal residues of the Rps $3 \mathrm{~N}$-domain. The orientation of the two molecules was not fixed in the calculations, allowing for variations in the overall structure of the complex. The obtained model showed a good agreement with the experimental data $\left(\gamma^{2}=1.056, \gamma=1.028\right.$ and $\chi^{2}$ free $\left.=0.996\right)$.

We then used the data with the Rps3 N-domain in complex with Yar1-FL Although we do not observe any interaction-induced folding of the Yar1 extensions, they are in a slightly more compact conformation than in the free Yarl, as evidenced by the smaller radius of gyration $(28.6 \AA)$. By using the models from the determined structures of Rps3 N-domain/Yar1-core and isolated Yar1-FL, an initial model was built and refined for all the unstructured regions. The refined model shows a good agreement with the experimental data $\left(\chi^{2}=1.380, \chi=1.175\right.$ and $\chi^{2}$ free $=1.306$ ). The core domains of the complex stay in the same conformation as in the crystal structure, and the unstructured regions adopt an unfolded and extended conformation.

Finally, for the model of the dimeric full-length Rps3/Yarl complex, initial models of the three possible dimers were built from the crystal structures, and completed with the unstructured regions determined in the SAXS model of the Rps3 N-domain/Yarl complex. All the extensions and relative molecule orientations were then optimized against the experimental data.

Purification of His6-tagged proteins. Expression constructs were cloned into a pETDuet-1 vector (Novagen) and transformed in an Escherichia coli BL21 (DE3) Rosetta Star strain. Cells were cultured in $\mathrm{LB}$ medium at $30^{\circ} \mathrm{C}$ to an $\mathrm{OD}_{600}$ of
0.3-0.4. Protein expression was induced with $0.3 \mathrm{mM}$ isopropyl $\beta$-D-thiogalactoside (IPTG), and cultures were shifted to $16^{\circ} \mathrm{C}$ for $20 \mathrm{~h}$. Cell pellets were resuspended in lysis buffer containing $50 \mathrm{mM}$ Tris (pH 7.5), $150 \mathrm{mM} \mathrm{NaCl}, 40 \mathrm{mM}$ imidazole, HP protease inhibitor mix (Serva), $0.5 \mathrm{mM}$ phenylmethylsulphonyl fluoride (PMSF), $1 \mathrm{mM}$ dithiothreitol (DTT) and $1 \mathrm{mg} \mathrm{ml}^{-1}$ lysozyme, and lysed by sonication. Cell lysates were centrifuged at $40,000 \mathrm{~g}$ at $4^{\circ} \mathrm{C}$ for $30 \mathrm{~min}$ to remove insoluble material, and supernatants were incubated for $1 \mathrm{~h}$ under rotation at $4{ }^{\circ} \mathrm{C}$ with Ni-NTA agarose (Qiagen). Beads were then washed three times with $\sim 10 \mathrm{ml}$ washing buffer (50 mM Tris (pH 7.5), $150 \mathrm{mM} \mathrm{NaCl}, 40 \mathrm{mM}$ imidazole and $1 \mathrm{mM} \mathrm{DTT}$ ), and bound material was subsequently eluted under rotation for $20 \mathrm{~min}$ at $4{ }^{\circ} \mathrm{C}$ with $300 \mathrm{mM}$ imidazole. When indicated in the respective figures, the obtained eluates were subjected to a second affinity purification step to pull down co-purified interaction partners with anti-Flag agarose (Sigma). After incubation for $1 \mathrm{~h}$ under rotation at $4{ }^{\circ} \mathrm{C}$, beads were washed four times with washing buffer $(50 \mathrm{mM}$ Tris (pH 7.5), $150 \mathrm{mM} \mathrm{NaCl}$ and $1 \mathrm{mM}$ DTT), and bound material was eluted with $100 \mu \mathrm{g} \mathrm{ml}^{-1}$ Flag-peptide (Sigma). Eluates were analysed by SDS-polyacrylamide gel electrophoresis (SDS-PAGE) using $12 \%$ or $4-12 \%$ polyacrylamide gels (Invitrogen), and protein bands were visualized by Coomassie staining or western blotting.

Purification of Flag-tagged proteins. Flag-Yarl was expressed from the pETDuet-1 vector (Novagen), Ltv1-Flag and Enp1-Flag-TEV-ProteinA (FPA) were expressed from the pET15b vector (Novagen) with the conditions described above. Cell pellets were resuspended in lysis buffer containing $50 \mathrm{mM}$ Tris ( $\mathrm{pH} 7.5$ ), $150 \mathrm{mM} \mathrm{NaCl}$, HP protease inhibitor mix (Serva), $0.5 \mathrm{mM}$ PMSF, $1 \mathrm{mM}$ DTT and $1 \mathrm{mg} \mathrm{ml}^{-1}$ lysozyme, and lysed by sonication. Cell lysates were centrifuged at $40,000 \mathrm{~g}$ and $4^{\circ} \mathrm{C}$ for $30 \mathrm{~min}$ to remove insoluble material, and supernatants were incubated for $1 \mathrm{~h}$ under rotation at $4{ }^{\circ} \mathrm{C}$ with anti-Flag agarose (Sigma). Beads were then washed three times with $\sim 10 \mathrm{ml}$ washing buffer $(50 \mathrm{mM}$ Tris $(\mathrm{pH} 7.5)$, $150 \mathrm{mM} \mathrm{NaCl}$ and $1 \mathrm{mM}$ DTT), and bound material was subsequently eluted under rotation for $45 \mathrm{~min}$ at $4{ }^{\circ} \mathrm{C}$ with $100 \mu \mathrm{g} \mathrm{ml}{ }^{-1}$ Flag-peptide (Sigma).

For the experiment in Fig. 7a, Flag-tagged Ltv1 truncations were co-expressed with His6-Rps20 from the pETDuet-1 vector and affinity-purified with anti-Flag agarose as described above. Lysis and washing buffers contained $400 \mathrm{mM} \mathrm{NaCl}$.

Purification of GST-TEV-Hrr25. GST-TEV-Hrr25 was expressed from the pProEx-1 vector ${ }^{43}$ after induction with $0.3 \mathrm{mM}$ IPTG for $16 \mathrm{~h}$ at $18^{\circ} \mathrm{C}$. Cell pellets were resuspended in lysis buffer containing $50 \mathrm{mM}$ Tris (pH 7.5), $150 \mathrm{mM} \mathrm{NaCl}$ 
HP protease inhibitor mix (Serva), $0.5 \mathrm{mM}$ PMSF, $1 \mathrm{mM} \mathrm{DTT}$ and $1 \mathrm{mg} \mathrm{ml}^{-1}$ lysozyme, and lysed by sonication. Cell lysates were centrifuged at $40,000 \mathrm{~g}$ and $4{ }^{\circ} \mathrm{C}$ for $30 \mathrm{~min}$ to remove insoluble material, and supernatants were incubated for $1 \mathrm{~h}$ under rotation at $4{ }^{\circ} \mathrm{C}$ with glutathione (GSH) agarose (Sigma). Beads were then washed three times with $\sim 10 \mathrm{ml}$ washing buffer (50 mM Tris ( $\mathrm{pH} 7.5), 150 \mathrm{mM}$ $\mathrm{NaCl}$ and $1 \mathrm{mM} \mathrm{DTT}$ ), and bound GST-TEV-Hrr25 was eluted under rotation for $1 \mathrm{~h}$ at room temperature with tobacco etch virus (TEV) protease.

In vitro phosphorylation assay. Affinity-purified Ltv1-Flag, Ltv1(S336A/S339A/ S342A)-Flag and Ltvl(S6A)-Flag were incubated with affinity-purified Hrr25 for $20 \mathrm{~min}$ at $30^{\circ} \mathrm{C}$ in a buffer containing $50 \mathrm{mM}$ Tris (pH 7.5), $150 \mathrm{mM} \mathrm{NaCl}, 5 \mathrm{mM}$ $\mathrm{MgCl}_{2}, 0.1 \mathrm{mM}$ ATP and $1 \mathrm{mM}$ DTT. Reactions were stopped by addition of SDS-PAGE loading buffer and boiling at $95^{\circ} \mathrm{C}$ for $10 \mathrm{~min}$. Samples were analysed on $4-12 \%$ SDS-polyacrylamide gels and Coomassie staining or western blotting with biotin-labelled Phos-tag (Wako Pure Chemical Industries) in combination with streptavidin-horseradish peroxidase (Life Technologies).

Tandem affinity purification. TAP purifications were performed as described previously ${ }^{44}$. Diploid strains used for experiments shown in Fig. 2 were grown at $25^{\circ} \mathrm{C}$, strains used for purifications shown in Supplementary Fig. 4a were grown at $30^{\circ} \mathrm{C}$. Cell pellets were resuspended in lysis buffer containing $50 \mathrm{mM}$ Tris ( $\left.\mathrm{pH} 7.5\right)$, $100 \mathrm{mM} \mathrm{NaCl}, 2 \mathrm{mM} \mathrm{MgCl} 2,0.075 \% \mathrm{NP}-40,1 \mathrm{mM}$ DTT, $0.5 \mathrm{mM}$ PMSF and $1 \times$ FY protease inhibitor mix (Serva), and lysed by mechanical disruption with glass beads. Lysates were cleared by centrifugation, mixed with IgG Sepharose 6 Fast Flow (GE Healthcare) and incubated at $4{ }^{\circ} \mathrm{C}$ for $90 \mathrm{~min}$. Beads were spinned down, washed with lysis buffer (without protease inhibitors) and transferred into Mobicol columns (MoBiTec). After washing with 20 column volumes, samples were eluted with TEV protease for $75 \mathrm{~min}$ at RT. After addition of $2 \mathrm{mM} \mathrm{CaCl}_{2}$, eluates were incubated with Calmodulin Sepharose 4B (GE Healthcare) for $1 \mathrm{~h}$ at room temperature. Beads were washed with 10 column volumes of lysis buffer containing $2 \mathrm{mM} \mathrm{CaCl}_{2}$ and eluted with buffer containing $5 \mathrm{mM}$ EGTA. For the experiments shown in Fig. 2, the obtained EGTA eluates were incubated with antiFlag agarose (Sigma) for $1 \mathrm{~h}$ at $4{ }^{\circ} \mathrm{C}$. Beads were washed with eight column volumes of lysis buffer, and bound proteins were subsequently eluted with $100 \mu \mathrm{g} \mathrm{ml}^{-1}$ Flag-peptide (Sigma). Final eluates were precipitated, resuspended in SDS sample buffer and loaded onto $12 \%$ (Fig. 2b,d) or 4-12\% (Supplementary Fig. 4a) NuPAGE Bis-Tris gels (Thermo Fisher).

Uncropped scans of Coomassie-stained gels and western blots in Fig. 2b,d are shown in Supplementary Fig. 8.

Sucrose gradient analysis. Cells were grown at $30^{\circ} \mathrm{C}$ in $100 \mathrm{ml}$ yeast extract peptone dextrose (YPD) medium to logarithmic growth phase $\left(A_{600}\right.$ of $\left.\sim 0.6\right)$. Approximately, $100 \mu \mathrm{g} \mathrm{ml}^{-1} \mathrm{CHX}$ was added to the cultures and after incubation for $5 \mathrm{~min}$ on ice, cells were pelleted and resuspended in lysis buffer $(10 \mathrm{mM}$ Tris$\mathrm{HCl}$ (pH 7.5), $100 \mathrm{mM} \mathrm{NaCl}, 30 \mathrm{mM} \mathrm{MgCl}_{2}$ and $100 \mu \mathrm{g} \mathrm{ml}^{-1} \mathrm{CHX}$ ). After cell lysis with glass beads, $5 A_{260}$ units of the cell extracts were loaded onto $7-45 \%$ sucrose gradients and centrifuged at $180,000 \mathrm{~g}$ for $2 \mathrm{~h} 45 \mathrm{~min}$ at $4{ }^{\circ} \mathrm{C}$. Gradients were analysed using a UA-6 system (Teledyne Isco) with continuous monitoring at $A_{254 \mathrm{~nm}}$.

Western blotting. Western blot analysis was performed using the following antibodies: anti-Yar1 antibody (1:5,000; ref. 22), anti-Rps3 antibody (1:30,000, provided by Matthias Seedorf), anti-Rps8 antibody (1:5,000, provided by Giorgio Dieci), anti-Rps2/Rpl30 antibody (1:2,000, provided by Jonathan Warner), antihRps5 antibody (1:500, Sigma-Aldrich, cat. no. HPA055878), anti-Rpl35 antibody (1:35,000, provided by Matthias Seedorf), anti-Rps26/Tsr2 antibody (1:2,000, provided by Vikram Panse), anti-Ltvl antibody (1:8,000, provided by Katrin Karbstein), anti-Enp1 antibody (1:4,000, provided by Katrin Karbstein), anti-Tsr1 antibody (1:4,000, provided by Katrin Karbstein), anti-Dim1 antibody (1:4,000, provided by Katrin Karbstein), anti-Rio2-antibody (1:500, Santa Cruz Biotechnology, cat. no. sc-98828), anti-CBP antibody (1:4,000, Merck-Millipore, cat. no. $07-482$ ), secondary anti-rabbit horseradish peroxidase-conjugated antibody (1:15,000, Sigma-Aldrich, cat. no. A0545), horseradish peroxidase-conjugated antiHis6 antibody (1:15,000, Sigma-Aldrich, cat. no. A7058), horseradish peroxidaseconjugated anti-Flag antibody (1:15,000, Sigma-Aldrich, cat. no. A8592).

The biotinylated phosphate-binding reagent Phos-Tag (Wako Pure Chemical Industries) was prepared according to manufacturer's instructions and used to detect protein phosphorylation in combination with streptavidin-HRP (Invitrogen)

Y2H interaction analyses. Plasmids expressing the bait proteins, fused to the GAL4 DNA-binding domain (G4BD), and the prey proteins, fused to the GAL4 activation domain (G4AD), were co-transformed into the reporter strain PJ69-4A. $\mathrm{Y} 2 \mathrm{H}$ interactions were documented by spotting representative transformants in 10 -fold serial dilution steps on synthetic dextrose complete (SDC)-Trp-Leu, SDC-Trp-Leu-His (HIS3 reporter) and SDC-Trp-Leu-Ade (ADE2 reporter) plates. Growth on SDC-Trp-Leu-His plates is indicative of a weak interaction, whereas only relatively strong interactions permit growth on SDC-Trp-Leu-Ade plates.
RNA isolation and northern blotting. Total RNA preparations were performed from $20 A_{600}$ units using the mechanical disruption protocol of the RNeasy mini kit (Qiagen). Approximately, $3 \mu \mathrm{g}$ of RNA per sample were separated on $1.5 \%$ MOPS-agarose gels as described in the manual for the RNeasy mini kit. The RNA was transferred overnight onto a Hybond-N nylon membrane (Amersham Biosciences), and then crosslinked to the membrane by ultraviolet radiation. Hybridization was performed overnight at $42^{\circ} \mathrm{C}$ in $500 \mathrm{mM} \mathrm{NaPO}_{4}$ buffer (pH 7.2), 7\% SDS and $1 \mathrm{mM}$ EDTA using $5^{\prime}-32$ P-labelled oligonucleotides with the following sequences: 20S, $5^{\prime}$-GACTCTCCATCTCTTGTCTTCTTG- $3^{\prime}$; 25S, $5^{\prime}$-CTCCGCTTATTGATATGC- $3^{\prime} ; 18 \mathrm{~S}, 5^{\prime}$-CATGGCTTAATCTTTGAGAC- ${ }^{\prime}$. The membranes were washed three times for $20 \mathrm{~min}$ at $42^{\circ} \mathrm{C}$ in $40 \mathrm{mM} \mathrm{NaPO}_{4}$ buffer ( $\mathrm{pH} 7.2)$ and $1 \% \mathrm{SDS}$, and radioactivity was detected by exposing X-ray films. Membranes were regenerated by washing in $1 \%$ SDS.

Fluorescence microscopy. Life yeast cells were imaged by fluorescence microscopy using a Zeiss Axioskop microscope. When indicated, $0.2 \mu \mathrm{g} \mathrm{ml}^{-1} \mathrm{LMB}$ and $20 \mu \mathrm{g} \mathrm{ml}^{-1} \mathrm{CHX}$ were added.

\section{References}

1. de la Cruz, J., Karbstein, K. \& Woolford, Jr J. L. Functions of ribosomal proteins in assembly of eukaryotic ribosomes in vivo. Annu. Rev. Biochem. 84, 93-129 (2015).

2. Henras, A. K. et al. The post-transcriptional steps of eukaryotic ribosome biogenesis. Cell. Mol. Life Sci. 65, 2334-2359 (2008).

3. Kressler, D., Hurt, E. \& Bassler, J. Driving ribosome assembly. Biochim. Biophys. Acta 1803, 673-683 (2010).

4. Woolford, Jr J. L. \& Baserga, S. J. Ribosome biogenesis in the yeast Saccharomyces cerevisiae. Genetics 195, 643-681 (2013).

5. Ferreira-Cerca, S. et al. ATPase-dependent role of the atypical kinase Rio2 on the evolving pre-40S ribosomal subunit. Nat. Struct. Mol. Biol. 19, 1316-1323 (2012).

6. Ferreira-Cerca, S., Kiburu, I., Thomson, E., LaRonde, N. \& Hurt, E. Dominant Riol kinase/ATPase catalytic mutant induces trapping of late pre-40S biogenesis factors in 80S-like ribosomes. Nucleic Acids Res. 42, 8635-8647 (2014).

7. Ghalei, H. et al. Hrr25/CK1 $\Delta$-directed release of Ltv1 from pre-40S ribosomes is necessary for ribosome assembly and cell growth. J. Cell Biol. 208, 745-759 (2015).

8. Schäfer, T. et al. Hrr25-dependent phosphorylation state regulates organization of the pre-40S subunit. Nature 441, 651-655 (2006).

9. Strunk, B. S. et al. Ribosome assembly factors prevent premature translation initiation by $40 \mathrm{~S}$ assembly intermediates. Science 333, 1449-1453 (2011).

10. Strunk, B. S., Novak, M. N., Young, C. L. \& Karbstein, K. A translation-like cycle is a quality control checkpoint for maturing $40 \mathrm{~S}$ ribosome subunits. Cell 150, 111-121 (2012).

11. Turowski, T. W. et al. Riol mediates ATP-dependent final maturation of $40 \mathrm{~S}$ ribosomal subunits. Nucleic Acids Res. 42, 12189-12199 (2014).

12. Widmann, B. et al. The kinase activity of human Riol is required for final steps of cytoplasmic maturation of 40S subunits. Mol. Biol. Cell 23, 22-35 (2012).

13. Wyler, E. et al. Tandem affinity purification combined with inducible shRNA expression as a tool to study the maturation of macromolecular assemblies. RNA 17, 189-200 (2011)

14. Zemp, I. et al. Distinct cytoplasmic maturation steps of $40 \mathrm{~S}$ ribosomal subunit precursors require hRio2. J. Cell Biol. 185, 1167-1180 (2009).

15. Zemp, I. et al. CK1 $\delta$ and CK1 1 are components of human 40 S subunit precursors required for cytoplasmic 40S maturation. J. Cell Sci. 127, 1242-1253 (2014).

16. Fatica, A., Oeffinger, M., Dlakić, M. \& Tollervey, D. Noblp is required for cleavage of the $3^{\prime}$ end of 18 SRNA. Mol. Cell. Biol. 23, 1798-1807 (2003).

17. Lamanna, A. C. \& Karbstein, K. Nob1 binds the single-stranded cleavage site D at the $3^{\prime}$-end of $18 \mathrm{~S}$ rRNA with its PIN domain. Proc. Natl Acad. Sci. USA 106, 14259-14264 (2009).

18. Pertschy, B. et al. RNA helicase Prp43 and its co-factor Pfal promote 20 to $18 \mathrm{~S}$ rRNA processing catalyzed by the endonuclease Nob1. J. Biol. Chem. 284, 35079-35091 (2009).

19. García-Gómez, J. J. et al. Final pre-40S maturation depends on the functional integrity of the 60S subunit ribosomal protein L3. PLoS Genet. 10, e1004205 (2014).

20. Lebaron, S. et al. Proofreading of pre- 40 S ribosome maturation by a translation initiation factor and 60S subunits. Nat. Struct. Mol. Biol. 19, 744-753 (2012).

21. Granneman, S., Petfalski, E., Swiatkowska, A. \& Tollervey, D. Cracking pre-40S ribosomal subunit structure by systematic analyses of RNA-protein crosslinking. EMBO J. 29, 2026-2036 (2010).

22. Koch, B. et al. Yar1 protects the ribosomal protein Rps3 from aggregation. J. Biol. Chem. 287, 21806-21815 (2012).

23. Holzer, S., Ban, N. \& Klinge, S. Crystal structure of the yeast ribosomal protein rpS3 in complex with its chaperone Yar1. J. Mol. Biol. 425, 4154-4160 (2013). 
24. Ben-Shem, A. et al. The structure of the eukaryotic ribosome at $3.0 \AA$ resolution. Science 334, 1524-1529 (2011).

25. Hector, R. D. et al. Snapshots of pre-rRNA structural flexibility reveal eukaryotic $40 \mathrm{~S}$ assembly dynamics at nucleotide resolution. Nucleic Acids Res. 42, 12138-12154 (2014)

26. Loar, J. W. et al. Genetic and biochemical interactions among Yarl, Ltv1 and Rps3 define novel links between environmental stress and ribosome biogenesis in Saccharomyces cerevisiae. Genetics 168, 1877-1889 (2004).

27. Merwin, J. R. et al. Genetic analysis of the ribosome biogenesis factor Ltv1 of Saccharomyces cerevisiae. Genetics 198, 1071-1085 (2014).

28. Flotow, H. et al. Phosphate groups as substrate determinants for casein kinase I action. J. Biol. Chem. 265, 14264-14269 (1990).

29. Ptacek, J. et al. Global analysis of protein phosphorylation in yeast. Nature 438, 679-684 (2005)

30. Lord, C. et al. Sequential interactions with Sec 23 control the direction of vesicle traffic. Nature 473, 181-186 (2011).

31. Petronczki, M. et al. Monopolar attachment of sister kinetochores at meiosis I requires casein kinase 1. Cell 126, 1049-1064 (2006)

32. Tanaka, C. et al. Hrr25 triggers selective autophagy-related pathways by phosphorylating receptor proteins. J. Cell Biol. 207, 91-105 (2014).

33. Pausch, P. et al. Co-translational capturing of nascent ribosomal proteins by their dedicated chaperones. Nat. Commun. 6, 7494 (2015).

34. Adilakshmi, T., Bellur, D. L. \& Woodson, S. A. Concurrent nucleation of $16 \mathrm{~S}$ folding and induced fit in 30 S ribosome assembly. Nature 455, 1268-1272 (2008).

35. Kim, H. et al. Protein-guided RNA dynamics during early ribosome assembly. Nature 506, 334-338 (2014).

36. Ferreira-Cerca, S. et al. Analysis of the in vivo assembly pathway of eukaryotic 40S ribosomal proteins. Mol. Cell 28, 446-457 (2007).

37. Yatime, L., Hein, K. L., Nilsson, J. \& Nissen, P. Structure of the RACK1 dimer from Saccharomyces cerevisiae. J. Mol. Biol. 411, 486-498 (2011).

38. David, G. \& Pérez, J. Combined sampler robot and high-performance liquid chromatography: a fully automated system for biological small-angle X-ray scattering experiments at the Synchrotron SOLEIL SWING beamline. J. Appl. Cryst. 42, 892-900 (2009).

39. Petoukhov, M. V. et al. New developments in the ATSAS program package for small-angle scattering data analysis. J. Appl. Crystallogr. 45, 342-350 (2012).

40. Šali, A. \& Blundell, T. L. Comparative protein modelling by satisfaction of spatial restraints. J. Mol. Biol. 234, 779-815 (1993).

41. Evrard, G., Mareuil, F., Bontems, F., Sizun, C. \& Perez, J. DADIMODO: a program for refining the structure of multidomain proteins and complexes against small-angle scattering data and NMR-derived restraints. J. Appl. Crystallogr. 44, 1264-1271 (2011).

42. Rambo, R. P. \& Tainer, J. A. Accurate assessment of mass, models and resolution by small-angle scattering. Nature 496, 477-481 (2013).

43. Lutzmann, M., Kunze, R., Buerer, A., Aebi, U. \& Hurt, E. Modular selfassembly of a Y-shaped multiprotein complex from seven nucleoporins. EMBO J. 21, 387-397 (2002)
44. Puig, O. et al. The tandem affinity purification (TAP) method: a general procedure of protein complex purification. Methods 24, 218-229 (2001).

\section{Acknowledgements}

We thank Giorgio Dieci, Katrin Karbstein, Vikram Panse, Matthias Seedorf and Jonathan Warner for the generous gift of antibodies; Ed Hurt for providing plasmids; and Ellen Zechner for critically reading the manuscript. We acknowledge SOLEIL and ESRF for provision of synchrotron radiation facilities; and we would like to thank the beamline scientists on the SWING and BM29 beamlines. The research was funded by grants P23682-B20, P23883-B20, P27996-B21 and V292-B20 from the Austrian Science Fund (FWF) to B.P., and grants PP00P3_123341, PP00P3_146344/1 and 31003A_156764/1 from the Swiss National Science Foundation (SNSF) to D.K. Work at UPD was supported by CNRS, University Paris Descartes, the RNPGenesis grant from the Agence Nationale de la Recherche (ANR JC RNPGenesis), and the Institut Universitaire de France.

\section{Author contributions}

V.M., G.M., N.L., D.K. and B.P. conceived the experiments. V.M., G.M., S.R., M.B., L.D., T.S., D.K. and B.P. carried out the experiments. V.M., G.M., T.S., D.K. and B.P. constructed strains and plasmids. V.M., G.M., S.R., M.B., L.D., N.L., D.K. and B.P. analysed the data. All authors discussed the results. V.M., N.L., D.K. and B.P. wrote the manuscript; and G.M., H.B., S.R. and T.S. commented on the manuscript.

\section{Additional information}

Accession codes: SAXS data have been deposited in the BIOISIS depository with the IDs YAR1P (Yar1 full-length protein), Y1R3DP (Yar1 core/Rps3 N-domain), YRNTRP (Yar1/Rps3 N-domain) and YFLRNP (Yar1/Rps3)

Supplementary Information accompanies this paper at http://www.nature.com/ naturecommunications

Competing financial interests: The authors declare no competing financial interests

Reprints and permission information is available online at http://npg.nature.com/ reprintsandpermissions/

How to cite this article: Mitterer, V. et al. Sequential domain assembly of ribosomal protein S3 drives 40S subunit maturation. Nat. Commun. 7:10336 doi: 10.1038/ncomms10336 (2016).

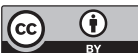

This work is licensed under a Creative Commons Attribution 4.0 International License. The images or other third party material in this article are included in the article's Creative Commons license, unless indicated otherwise in the credit line; if the material is not included under the Creative Commons license, users will need to obtain permission from the license holder to reproduce the material. To view a copy of this license, visit http://creativecommons.org/licenses/by/4.0/ 